\title{
Spectral theory of a class of nilmanifolds attached to Clifford modules
}

\author{
Wolfram Bauer $^{1} \cdot$ Kenro Furutani $^{2} \cdot$ Chisato Iwasaki $^{3} \cdot$ Abdellah Laaroussi $^{1}$
}

Received: 4 November 2019 / Accepted: 19 February 2020 / Published online: 3 April 2020

(c) The Author(s) 2020

\begin{abstract}
We determine the spectrum of the sub-Laplacian on pseudo $H$-type nilmanifolds and present pairs of isospectral but non-homeomorphic nilmanifolds with respect to the sub-Laplacian. We observe that these pairs are also isospectral with respect to the Laplacian. More generally, our method allows us to construct an arbitrary number of isospectral but mutually nonhomeomorphic nilmanifolds. Finally, we present two nilmanifolds of different dimensions such that the short time heat trace expansions of the corresponding sub-Laplace operators coincide up to a term which vanishes to infinite order as time tends to zero.
\end{abstract}

Keywords Sub-Laplacian · Subriemannian manifold · Isospectral · Heat kernel $\cdot$ Pseudo $H$-type group

Mathematics Subject Classification 58J53 $\cdot 58 \mathrm{~J} 50$

The first and the last named author have been supported by the priority program SPP 2026 geometry at infinity of Deutsche Forschungsgemeinschaft (project number BA 3793/6-1), the second named author was supported by the Grant-in-aid for Scientific Research (C) No. 17K05284, JSPS; the third named author was supported by the Grant-in-aid for Scientific Research (C) No. 24540189, JSPS.

Wolfram Bauer

bauer@math.uni-hannover.de

Kenro Furutani

furutani_kenro@ma.noda.tus.ac.jp

Chisato Iwasaki

iwasaki@sci.u-hyogo.ac.jp

Abdellah Laaroussi

abdellah.laaroussi@math.uni-hannover.de

1 Institut für Analysis, Welfengarten 1, 30167 Hannover, Germany

2 Advanced Mathematical Institute, Osaka City University, 3-3-138 Sugimoto, Sumiyoshi-ku, Osaka City 558-8585, Japan

3 Department of Mathematics, School of Science, University of Hyogo, 2167 Shosha Himeji, Hyogo 671-2201, Japan 


\section{Introduction}

In 1966 Mark Kac's famous paper [22] asked the question "Can one hear the shape of a drum?". This work can be regarded as the beginning of a central topic of spectral geometry although the problem itself traces back to Hermann Weyl's work at the beginning of the 20th century. Especially in the multi-dimensional situation, a negative answer to the above question was expected early on. Therefore, an important task was to construct isospectral but nonisometric or non-diffeomorphic or even non-homeomorphic manifolds. Such examples allow to determine geometric properties that are not determined by the spectrum. In high dimensions the first example of such manifolds was given by Milnor even earlier in 1964. In [32] a pair of 16-dimensional flat tori have been constructed which are isospectral but non-isometric. Nowadays, a general construction method by Sunada [37] and a wide range of examples are known, cf. [16,17,19,20,27]. In particular, they include lens spaces, spherical space forms or Heisenberg manifolds. Generalizing the last example the isospectrality problem may be considered for quotients $\Gamma \backslash \mathbb{G}$ of nilpotent Lie groups $\mathbb{G}$ of step $k \geq 2$ by a lattice $\Gamma$. In the following we will call such manifolds $k$-step nilmanifolds. Via an adaptation of representation theoretical methods due to C.S. Gordon and N.E. Wilson, pairs of isospectral nilmanifolds $\left(\Gamma_{1} \backslash \mathbb{G}, \Gamma_{2} \backslash \mathbb{G}\right)$ of step $k \geq 3$ were constructed in [17]. Different from the known examples based on Sunada's theorem these manifolds need not to be isospectral for the Laplacian on 1 -forms.

In the realm of Riemannian geometry it remains an interesting problem to construct isospectral but non-homeomorphic manifolds in a systematic way. Moreover, by restricting M. Kac's question to specific sub-classes of smooth manifolds (e.g. spheres or certain nilmanifolds) or by considering the spectrum of geometric operators different from the Laplacian one is led to new classification problems.

In the present paper we consider Kac's question for a class of subriemannian manifolds $M$ carrying a geometrically defined second order sub-elliptic differential operator, called sub-Laplacian. More precisely, in our setup $M$ is assumed to be a nilmanifold of step 2 whose covering simply connected nilpotent Lie group is of pseudo $H$-type. Such groups are generalizations of the well known Heisenberg type groups introduced by A. Kaplan in [23]. Their Lie algebras are called of pseudo $H$-type as well and were first considered in [10]. Pseudo $H$-type Lie algebras are constructed from Clifford algebras $C \ell_{r, s}$ of signature $(r, s)$ and their (admissible) modules, cf. Sect. 4 or [10,13-15] for a definition and more details. We also recall that the existence of lattices $\Gamma$ in pseudo $H$-type Lie groups $\mathbb{G}$ has been proven in [13]. With respect to a standard (integral) lattice we can therefore consider compact left-coset spaces $\Gamma \backslash \mathbb{G}$.

Based on an explicit heat trace formula for the sub-Laplacian combined with the recent classification of pseudo $H$-type algebras in $[14,15]$ we can give the negative answer to Kac's question in this non-standard setting and present a list of new examples. The subriemannian structure we deal with naturally extends to a Riemannian structure and we may as well consider the corresponding Laplacian $\Delta$ on $M$. As it turns out in our examples the difference $D:=\Delta-\Delta_{\text {sub }}$ is a "sum-of-squares-operator", i.e. it can be expressed in the form

$$
D=-\frac{1}{2} \sum_{k} Z_{k}^{2}
$$

with globally defined vector fields $Z_{k}$ on $M$. Moreover, the operators $D$ and $\Delta_{\text {sub commute }}$ and therefore $\Delta$ and $\Delta_{\text {sub }}$ commute as well. As a consequence we have obtained new exam- 
ples of isospectral, non-homeomorphic manifolds in the usual sense, i.e. with respect to the Riemannian structure.

Different from previously studied isospectral, non-isometric quotients $\left(\Gamma_{1} \backslash \mathbb{G}, \Gamma_{2} \backslash \mathbb{G}\right)$ for which the covering simply connected Lie group $\mathbb{G}$ is fixed and the lattice $\Gamma$ varies we note that in our construction also the group $\mathbb{G}$ is varying in the definition of both manifolds. Furthermore, by choosing the space dimension suitably high our method not only allows us to select pairs but any given number of isospectral, non-homeomorphic nilmanifolds. To our best knowledge these are the first examples of this kind.

Before stating the results more in detail we review some definitions. By a subriemannian manifold we understand a triple $(M, \mathcal{H},\langle\cdot, \cdot\rangle)$ where $M$ is a smooth manifold (orientable and without boundary), $\mathcal{H}$ is a bracket generating subbundle in the tangent bundle $T M$ and $\langle\cdot, \cdot\rangle$ denotes a family of inner products on $\mathcal{H}$ which smoothly vary with the base point. Recall that $\mathcal{H}$ is called bracket generating if vector fields taking values in $\mathcal{H}$ together with a finite number of their iterated brackets span the tangent space at any point of $M$.

Based on the bracket generating condition one can assign to each point $q \in M$ a flag

$$
\mathcal{H}_{q}=\mathcal{H}_{q}^{1} \subset \mathcal{H}_{q}^{2} \subset \cdots \subset \mathcal{H}_{q}^{k(q)}=T_{q} M
$$

of vector spaces which exhausts the full tangent space (see [1] for details). The subriemannian structure is called equi-regular, if $k(q)$ and the dimensions $\operatorname{dim} \mathcal{H}_{q}^{j}$ for $j=1, \ldots, k(q)$ are independent of $q \in M$. Under this assumption and based on the Popp measure construction one intrinsically can define a sub-elliptic operator $\Delta_{\text {sub }}$ on $M$ which generalizes the LaplaceBeltrami operator in Riemannian geometry (cf. [1]). We call this operator sub-Laplacian whereas it is called intrinsic hypoelliptic Laplacian in [1]. In case of a left-invariant subriemannian structure on a unimodular Lie group, which includes the case of a nilpotent Lie group, this operator is known to be a sum-of-squares of vector fields, [1, Proposition 12]. The same remains true if we descend $\Delta_{\text {sub }}$ to the quotient of $G$ by a cocompact discrete subgroup (lattice) $\Gamma$ and consider the (intrinsic) sub-Laplacian on the left-coset space $M=\Gamma \backslash G$.

The manifolds $M$ in this paper are equipped with a bracket generating subbundle $\mathcal{H}$ which is trivial as a vector bundle and a metric on $\mathcal{H}$ which naturally extends to a Riemannian metric on $M$. More precisely, there is a globally defined frame $\left\{X_{i}\right\}$ of $\mathcal{H}$ which is orthonormal at any point and skew-symmetric with respect to a naturally chosen volume form such that ${ }^{1}$ :

$$
\Delta_{\text {sub }}=-\frac{1}{2} \sum_{i} X_{i}{ }^{2} .
$$

As is well-known the bracket generating property (also called Hörmander condition) implies that $\Delta_{\text {sub }}$ is a sub-elliptic operator (i.e. it satisfies an "a priori estimate with a loss of derivative", cf. [18]). Clearly, this property does not depend on the chosen Riemannian metric. As a consequence it can be shown that the sub-Laplacian on a closed manifold $M$ has a compact resolvent and spectrum only consisting of eigenvalues with finite multiplicities (see [18]). Hence we can define the notion of isospectrality of two given subriemannian manifolds by replacing the spectrum of the Laplacian with the spectrum of the sub-Laplacian.

The asymptotic distribution of eigenvalues for classes of self-adjoint operators with double characteristics acting on compact manifolds or an asymptotic expansion of the heat kernel for "sum-of-squares operators" with first order term satisfying Hörmander's condition have been obtained in $[7,8,21,29-31,36]$, respectively.

\footnotetext{
${ }^{1}$ In oder to simplify the heat kernel expression we have chosen the factor $\frac{1}{2}$ in front of the sum.
} 
In the special case where $M$ is a 2-step nilmanifold it was shown in [3] that the heat trace of the sub-Laplacian admits an asymptotic expansion similar to the heat trace expansion of the Laplacian on a torus.

In order to detect isospectral (subriemannian) nilmanifolds we first need to determine the spectrum of the sub-Laplacian $\Delta_{\text {sub }}^{\Gamma \backslash \mathbb{G}}$ on a 2-step nilmanifold $M=\Gamma \backslash \mathbb{G}$. Based on an explicit expression of the heat kernel for $\Delta_{\text {sub }}$ on the covering group $\mathbb{G}$ in $[6,9,12]$ a formula for the heat trace of $\Delta_{\text {sub }}^{\Gamma \backslash \mathbb{G}}$ descended from $\mathbb{G}$ to $M=\Gamma \backslash \mathbb{G}$ was obtained in [4]. In case of a pseudo $H$-type group $\mathbb{G}$ this trace formula simplifies further and in principle can be used to explicitly calculate the spectrum of $\Delta_{\text {sub }}$ on $M$. However, we need not to perform the full calculation. In order to identify isospectral manifolds it is sufficient to compare the corresponding trace formulas.

In a second step we need to classify non-homeomorphic nilmanifolds $\Gamma_{1} \backslash \mathbb{G}_{1}$ and $\Gamma_{2} \backslash \mathbb{G}_{2}$ of the same dimension. First, we reduce this task to a classification of pseudo $H$-type Lie algebras up to isomorphisms (cf. Corollary 7.2). Then we apply the very recent classification results in $[14,15]$.

The paper is organized as follows: in Sect. 2 we introduce the sub-Laplacian on a general 2-step nilpotent Lie group $\mathbb{G}$ and we recall an explicit integral expression of its heat kernel known as Beals-Gaveau-Greiner formula, cf. [5,6,9].

Assuming the existence of a lattice $\Gamma$ in $\mathbb{G}$ we decompose the sub-Laplacian $\Delta_{\text {sub }}$ on the compact nilmanifold $M=\Gamma \backslash \mathbb{G}$ into an infinite sum of elliptic operators acting on line bundles in Sect. 3. Via this method we obtain a decomposition of the heat trace of $\Delta_{\text {sub }}^{\Gamma \backslash \mathbb{G}}$ into the heat traces of its component elliptic operators, cf. [4], and we present a trace formula for the sub-Laplacian on $M$.

In Sect. 4 we recall the notion of pseudo $H$-type Lie algebras and groups following $[10,13,14]$. We discuss the existence and some basic properties of integral lattices for such groups. These will play a role in our construction in Sect. 7.

In Sect. 5 we study the eigenvalues of a matrix-valued function which encodes the structure constants of the pseudo $H$-type Lie algebra. These data are essential in the calculation of the heat kernel of the sub-Laplacian in Sect. 2 and the trace formula in Sect. 3. Based on the trace formula we give a criterion for isospectrality of two pseudo $H$-type nilmanifolds in Sect. 6 (Theorem 6.3).

The last sections contain our main results. We use the classification of pseudo $H$-type Lie algebras in $[14,15]$ to construct finite families of isospectral, non-homeomorphic pseudo $H$-type nilmanifolds. Finally, we present two nilmanifolds of different dimensions such that the short time heat trace expansions of the corresponding sub-Laplace operators coincide up to a term vanishing to infinite order as time tends to zero.

\section{Heat kernel on two step nilpotent Lie groups}

We recall the integral form of the heat kernel for a sub-Laplacian on simply connected two step nilpotent Lie groups given in [5,6], see also [9,12].

\subsection{Sub-Laplacian on two step nilpotent groups}

Let $\mathbb{G}$ be a simply connected two step nilpotent Lie group with Lie algebra $\mathcal{N}$. We assume that

$$
[\mathcal{N}, \mathcal{N}]=\text { center of } \mathcal{N}
$$


and we fix a basis $\left\{X_{i}, Z_{k} \mid i=1, \ldots, N, k=1, \ldots, d\right\}$ of $\mathcal{N}$ such that $\left\{Z_{k}\right\}_{k=1}^{d}$ and $\left\{X_{i}\right\}_{i=1}^{N}$ span the center $[\mathcal{N}, \mathcal{N}]$ and its complement, respectively. Moreover, we assume that $\mathcal{N}$ is equipped with an inner product with respect to which $\left\{X_{i}, Z_{k}\right\}$ becomes an orthonormal basis. Hence the Lie algebra $\mathcal{N}$ is decomposed into an orthogonal sum

$$
\mathcal{N}=\operatorname{span}\left\{X_{1}, \ldots, X_{N}\right\} \oplus \perp[\mathcal{N}, \mathcal{N}] \cong \mathbb{R}^{N} \oplus_{\perp} \mathbb{R}^{d} .
$$

The expansion of Lie brackets

$$
\left[X_{i}, X_{j}\right]=\sum_{k=1}^{d} c_{i j}^{k} Z_{k}
$$

defines the structure constants $c_{i j}^{k}=-c_{j i}^{k}$. Given $z=\sum_{k=1}^{d} z_{k} Z_{k} \in[\mathcal{N}, \mathcal{N}]$ we denote by $\Omega(z)$ the skew-symmetric matrix

$$
\Omega(z)=\sum_{k=1}^{d} z_{k}\left(c_{i j}^{k}\right) \in \mathbb{R}(N)=\text { : algebra of } N \times N \text { real matrices } .
$$

Remark 2.1 Throughout the paper we identify the group $\mathbb{G}$ with $\mathbb{R}^{N} \times \mathbb{R}^{d}$ via the above coordinates, i.e.

$$
\mathbb{G} \ni g=\sum_{i=1}^{N} x_{i} X_{i}+\sum_{k=1}^{d} z_{k} Z_{k} \longleftrightarrow\left(x_{1}, \ldots, x_{N}, z_{1}, \ldots, z_{d}\right) \in \mathbb{R}^{N} \times \mathbb{R}^{d} \cong \mathcal{N} .
$$

Then the exponential map exp : $\mathcal{N} \cong \mathbb{\Xi}$ is the identity. Via the Baker-Campbell Hausdorff formula and this identification we can express the group product $*$ on $\mathbb{G} \cong \mathcal{N}$ in the form

$$
g * h=g+h+\frac{1}{2}[g, h] .
$$

More explicitly and with respect to the above coordinates one has:

$$
\begin{aligned}
g * h & =\left(x_{1}, \ldots, x_{N}, z_{1}, \ldots, z_{d}\right) *\left(x_{1}^{\prime}, \ldots, x_{N}^{\prime}, z_{1}^{\prime}, \ldots, z_{d}^{\prime}\right) \\
& =\left(x_{1}+x_{1}^{\prime}, \ldots, x_{N}+x_{N}^{\prime}, z_{1}+z_{1}^{\prime}+\frac{1}{2} \sum_{i, j} c_{i j}^{1} x_{i} x_{j}^{\prime}, \ldots, z_{d}+z_{d}^{\prime}+\frac{1}{2} \sum_{i, j} c_{i j}^{d} x_{i} x_{j}^{\prime}\right) .
\end{aligned}
$$

Let $\widetilde{X}_{i}$ denote the left-invariant vector field on $\mathbb{G}$ corresponding to $X_{i} \in \mathcal{N}$ and consider the sub-Laplacian

$$
\Delta_{\mathrm{sub}}^{\mathbb{G}}=-\frac{1}{2} \sum_{i=1}^{N} \tilde{X}_{i}^{2} .
$$

Based on (2.1) the operator $\Delta_{\text {sub }}^{\mathbb{G}}$ is known to be sub-elliptic [18] and essentially selfadjoint in $L_{2}(\mathbb{G})$ with respect to the Haar measure and considered on compactly supported smooth functions $C_{0}^{\infty}(\mathbb{G})$, cf. [34,35].

\subsection{Beals-Gaveau-Greiner formula}

Next we recall the integral expression of the kernel function $K(t, g, h) \in C^{\infty}\left(\mathbb{R}_{+} \times \mathbb{G} \times \mathbb{G}\right)$ of the heat operator

$$
e^{-t \Delta_{\text {sub }}^{\mathbb{G}}}
$$


where $\mathbb{G}$ is a general 2-step nilpotent Lie group as above. The existence of a smooth kernel has been shown in $[34,35]$ and since the sub-Laplacian $\Delta_{\text {sub }}^{\mathbb{G}}$ is a left-invariant operator it follows that $K$ is a convolution kernel, i.e.

$$
K^{\mathbb{G}}(t, g, h)=k^{\mathbb{G}}\left(t, g^{-1} * h\right)
$$

with a smooth function $k^{\mathbb{G}} \in C^{\infty}\left(\mathbb{R}_{+} \times \mathbb{G}\right)$.

In $[5,6,9,12]$ an integral expression of $k^{\mathbb{G}}$ is given explicitly. Below we will calculate the spectrum of the sub-Laplacian on a class of nilmanifolds by using this expression. Recall that in the integrand of $k^{\mathbb{G}}$ two functions (action and volume function) appear. The integration is taken over a space which can be interpreted as the characteristic variety of the sub-Laplacian. Here we will neither present the details of this structure nor a proof of the next theorem.

Theorem 2.2 (Beals-Gaveau-Greiner formula, [5,9]) The integral kernel of the heat operator (2.5) has the form:

$$
K^{\mathbb{G}}(t, g, h)=k^{\mathbb{G}}\left(t, g^{-1} * h\right)=\frac{1}{(2 \pi t)^{N / 2+d}} \int_{\mathbb{R}^{d}} e^{-\frac{f\left(\tau, g^{-1} * h\right)}{t}} W(\tau) d \tau,
$$

where the functions $f=f(\tau, g) \in C^{\infty}\left(\mathbb{R}^{d} \times \mathbb{G}\right)$ and $W(\tau) \in C^{\infty}\left(\mathbb{R}^{d}\right)$ are given as follows: put $g=(x, z) \in \mathbb{R}^{N} \times \mathbb{R}^{d}$, then

$$
\begin{aligned}
f(\tau, g) & =f(\tau, x, z)=\sqrt{-1}\langle\tau, z\rangle+\frac{1}{2}\langle\Omega(\sqrt{-1} \tau) \operatorname{coth}(\Omega(\sqrt{-1} \tau)) \cdot x, x\rangle, \\
W(\tau) & =\left\{\operatorname{det} \frac{\Omega(\sqrt{-1} \tau)}{\sinh \Omega(\sqrt{-1} \tau)}\right\}^{1 / 2},
\end{aligned}
$$

where $\left\langle z, z^{\prime}\right\rangle=\sum_{k=1}^{d} z_{k} z_{k}^{\prime}$ denotes the Euclidean inner product on $\mathbb{R}^{d}$.

Remark 2.3 Later on we will use the notation $\langle\bullet, \bullet\rangle_{r, s}$ for a non-degenerate indefinite scalar product with the signature $(r, s)$ such that $\langle\bullet, \bullet\rangle=\langle\bullet, \bullet\rangle_{d, 0}$.

We call $f=f(\tau, x, z)$ and $W(\tau) d \tau$ the complex action function and the volume form, respectively. Recall that $f$ is constructed by the complex Hamilton-Jacobi method, and the volume function $W(\tau)$ is sometimes referred to as van Vleck determinant. It is the Jacobian of the correspondence between the space of initial conditions and boundary conditions when we solve the Hamilton equation associated to the symbol of the sub-Laplacian. The solution can be interpreted as the bi-characteristic flow in the subriemannian setting. We recall that the volume function satisfies a transport equation.

\section{Lattices and decomposition of a sub-Laplacian}

Based on Theorem 2.2 we describe the heat kernel of the sub-Laplacian descended to the quotient space $\Gamma \backslash \mathbb{G}$ (left coset space) by a lattice $\Gamma$. Such a space is called a (compact) 2-step nilmanifold. In the following we assume that there exists a lattice (cocompact discrete subgroup) in $\mathbb{G}$. We recall Malcev's Theorem:

Theorem 3.1 (Malćev, [26,33]) A nilpotent Lie group $G$ possesses a lattice $\Gamma$, i.e. $\Gamma \backslash G$ is compact, if and only if there exists a basis $\left\{Y_{i}\right\}$ in its Lie algebra $\mathfrak{g}$ such that the structure constants $\left\{\alpha_{i j}^{k}\right\}$ defined by

$$
\left[Y_{i}, Y_{j}\right]=\sum_{k} \alpha_{i j}^{k} Y_{k}
$$


are all rational numbers.

\subsection{Torus bundle and a family of elliptic operators}

We recall a heat trace formula which previously has been obtained in [4, Theorem 4.2]. Our analysis is essential based on this formula and in order to keep the paper self-contained we now repeat the main steps of the calculation.

Let $\Gamma$ be a lattice in a simply connected 2-step nilpotent Lie group $\mathbb{G} \cong \mathbb{R}^{N} \times \mathbb{R}^{d}$. The quotient space $\Gamma \backslash \mathbb{G}$ can be equipped with a subriemannian structure naturally inherited from that of $\mathbb{G}$. Its sub-Laplacian, which we now denote by $\Delta_{\text {sub }}^{\Gamma \backslash \mathbb{G}}$, is the operator descended from the sub-Laplacian $\Delta_{\text {sub }}^{\mathbb{G}}$ on $\mathbb{G}$.

For an element $g \in \mathbb{G}$ we will denote by $[g] \in \Gamma \backslash \mathbb{G}$ the corresponding class in the quotient space. Then, the heat kernel

$$
K^{\Gamma \backslash \mathbb{G}}(t,[g],[h]) \in C^{\infty}\left(\mathbb{R}_{+} \times \Gamma \backslash \mathbb{G} \times \Gamma \backslash \mathbb{G}\right)
$$

of the sub-Laplacian $\Delta_{\text {sub }}^{\Gamma \backslash \mathbb{G}}$ on the nilmanifold $\Gamma \backslash \mathbb{G}$ is given by

$$
\begin{aligned}
K^{\Gamma \backslash \mathbb{G}}(t,[g],[h]) & =\sum_{\gamma \in \Gamma} K^{\mathbb{G}}(t, \gamma * g, h) \\
& =\sum_{\gamma \in \Gamma} k^{\mathbb{G}}\left(t, g^{-1} * \gamma * h\right) \in C^{\infty}\left(\mathbb{R}_{+} \times \Gamma \backslash \mathbb{G} \times \Gamma \backslash \mathbb{G}\right) .
\end{aligned}
$$

Assuming the existence of a lattice $\Gamma$ in $\mathbb{G}$ we can decompose the sub-Laplacian into a family of differential operators acting on invariant subspaces according to a torus bundle structure of $\Gamma \backslash \mathbb{G}$. Next, we present some details and give the heat kernel expression for each component elliptic operator.

Let $\mathbb{A} \cong \mathbb{R}^{d}$ be the center of the group $\mathbb{G}$ where as before the identification is done with respect to the fixed orthonormal basis $\left\{Z_{k}\right\}$ of $\mathbb{A}$. We obtain a principal bundle with the structure group $\mathbb{A} /(\Gamma \cap \mathbb{A}) \cong \mathbb{T}^{\operatorname{dim} \mathbb{A}}=\mathbb{T}^{d}$

$$
\Gamma \backslash \mathbb{G} \longrightarrow(\Gamma / \Gamma \cap \mathbb{A}) \backslash(\mathbb{G} / \mathbb{A}) \cong(\Gamma * \mathbb{A}) \backslash \mathbb{G} .
$$

Note that the base space $(\Gamma / \Gamma \cap \mathbb{A}) \backslash(\mathbb{G} / \mathbb{A}) \cong(\Gamma * \mathbb{A}) \backslash \mathbb{G}$ is also a torus of dimension $\operatorname{dim} \mathbb{G}-\operatorname{dim} \mathbb{A}=N+d-d=N$. Since $\mathbb{A}$ is abelian, the subgroup $\Gamma * \mathbb{A}$ coincides with $\Gamma+\mathbb{A}$, i.e. with the sum in the Lie algebra.

Let $\mathbf{n}$ be an element in the "dual lattice" $[\Gamma \cap \mathbb{A}]^{*}$ of $\Gamma \cap \mathbb{A}$, that is, $\mathbf{n}$ is a linear functional on $\mathbb{A}$ with the property that

$$
\mathbf{n}(\gamma) \in \mathbb{Z} \text { for all } \gamma \in \Gamma \cap \mathbb{A} .
$$

We may express $\mathbf{n}$ in the form $\mathbf{n}=\sum_{k=1}^{d} n_{k} Z_{k}$ with integer coefficients $n_{k} \in \mathbb{Z}$ such that

$$
\mathbf{n}(\gamma)=\langle\mathbf{n}, \gamma\rangle=\sum n_{k}\left\langle Z_{k}, \gamma\right\rangle \in \mathbb{Z} \text { for all } \gamma \in \Gamma \cap \mathbb{A} \text {. }
$$

Then, the function space $C^{\infty}(\Gamma \backslash \mathbb{G})$ is decomposed via a Fourier series expansion:

$$
C^{\infty}(\Gamma \backslash \mathbb{G}) \ni^{\forall} f ; f(g)=\sum_{\mathbf{n} \in[\Gamma \cap \mathbb{A}]^{*}} \int_{\mathbb{T}^{d}} f(g * \lambda) \overline{\chi_{\mathbf{n}}(\lambda)} d \lambda,
$$


where $\chi_{\mathbf{n}}: \mathbb{T}^{d} \cong \mathbb{A} /(\Gamma \cap \mathbb{A}) \rightarrow U(1)$ with $\chi_{\mathbf{n}}(\lambda)=e^{2 \pi \sqrt{-1}\langle\mathbf{n}, \lambda\rangle}$ is a unitary character corresponding to a dual element $\mathbf{n} \in[\Gamma \cap \mathbb{A}]^{*}$. So, we decompose

$$
C^{\infty}(\Gamma \backslash \mathbb{G})=\sum_{\mathbf{n} \in[\Gamma \cap \mathbb{A}]^{*}} \mathcal{F}^{(\mathbf{n})},
$$

where

$$
\mathcal{F}^{(\mathbf{n})}=\left\{\int_{\mathbb{T}^{d}} f(g * \lambda) \overline{\chi_{\mathbf{n}}(\lambda)} d \lambda \mid f \in C^{\infty}(\Gamma \backslash \mathbb{G})\right\}
$$

The subspace $\mathcal{F}^{(\mathbf{n})}$ can be seen as a space of smooth sections of a line bundle $E^{(\mathbf{n})}$ on the base space $(\Gamma+\mathbb{A}) \backslash \mathbb{G} \cong(\Gamma / \Gamma \cap \mathbb{A}) \backslash(\mathbb{G} / \mathbb{A})$ associated to the character $\chi_{\mathbf{n}}$. The sub-Laplacian leaves invariant each subspace $\mathcal{F}^{(\mathbf{n})}$ and therefore it can be interpreted as a differential operator $\mathcal{D}^{(\mathbf{n})}$ acting on the line bundle $E^{(\mathbf{n})}$. Since the subbundle spanned by the (left)-invariant vector fields $\left\{\widetilde{X}_{i} \mid i=1, \ldots, N\right\}$ defines a connection, i.e., its linear span is equivariant and transversal to the structure group action by $\mathbb{A} /(\Gamma \cap \mathbb{A})$, each operator $\mathcal{D}^{(\mathbf{n})}$ is elliptic. Hence the sub-Laplacian $\Delta_{\text {sub }}^{\Gamma \backslash \mathbb{G}}$ can be seen as an infinite sum of elliptic operators on the torus $\Gamma * \mathbb{A} \backslash \mathbb{G}$.

As a consequence we obtain a decomposition of the operator trace:

$$
\operatorname{tr}\left(e^{-\Delta_{\text {sub }}^{\Gamma \backslash \mathbb{G}}}\right)=\sum_{\mathbf{n} \in[\Gamma \cap \mathbb{A}]^{*}} \operatorname{tr}\left(e^{-t \mathcal{D}^{(\mathbf{n})}}\right)
$$

Recall that $\left\{Z_{k} \mid k=1, \ldots, d\right\}$ denotes an orthonormal basis of the center $[\mathcal{N}, \mathcal{N}]$ of $\mathcal{N}$. As before we write $\widetilde{Z}_{k}, k=1, \ldots, d$ for the corresponding left-invariant vector fields on the group $\mathbb{G}$. We equip $\mathbb{G}$ with a left-invariant Riemannian metric defined by assuming that the frame $\left[\widetilde{X}_{1}, \ldots, \widetilde{X}_{N}, \widetilde{Z}_{1}, \ldots, \widetilde{Z}_{d}\right]$ is orthonormal at any point of $\mathbb{G}$. Then the corresponding Laplacian has the form

$$
\Delta^{\mathbb{G}}=\Delta_{\text {sub }}^{\mathbb{G}}-\frac{1}{2} \sum_{k=1}^{d} \widetilde{Z}_{k}^{2}
$$

The action of the difference $\Delta^{\mathbb{G}}-\Delta_{\text {sub }}^{\mathbb{G}}$ on the subspace $\mathcal{F}^{(\mathbf{n})}$ for each dual element $\mathbf{n} \in$ $[\Gamma \cap \mathbb{A}]^{*}$ is given as follows:

Proposition 3.2 Let $f \in \mathcal{F}^{(\mathbf{n})}$, then

$$
\left(\Delta^{\mathbb{G}}-\Delta_{\text {sub }}^{\mathbb{G}}\right) f=-\frac{1}{2} \sum_{k=1}^{d} \widetilde{Z}_{k}^{2}(f)=2 \pi^{2} \sum_{k=1}^{d} n_{k}^{2} \cdot f .
$$

\subsection{Heat trace of the component operators}

Next we give an expression of the heat trace of each operator $\mathcal{D}^{(\mathbf{n})}$. Recall that the heat kernel $K^{\Gamma \backslash \mathbb{G}}$ of $\Delta_{\text {sub }}^{\Gamma \backslash \mathbb{G}}$ is given by (3.1). Let $\mathcal{F}_{\Gamma}$ and $\mathcal{F}_{\Gamma \cap \mathbb{A}}$ be a fundamental domain for the lattice $\Gamma$ in $\mathbb{G}$ and $\Gamma \cap \mathbb{A}$ in the Euclidean space $\mathbb{A}$, respectively. Then the integral

$$
k_{\mathcal{D}^{(\mathbf{n})}}(t,[g],[h])=\int_{\mathcal{F}_{\Gamma \cap \mathbb{A}}} K^{\Gamma \backslash \mathbb{G}}(t,[g],[h] * \lambda) \overline{\chi_{\mathbf{n}}(\lambda)} d \lambda
$$


is the kernel function for the heat operator $e^{-t \mathcal{D}^{(\mathbf{n})}}$, that is it satisfies

$$
\begin{aligned}
& k_{\mathcal{D}^{(\mathbf{n})}}(t,[g] * \theta,[h])=k_{\mathcal{D}^{(\mathbf{n})}}(t,[g * \theta],[h])=\overline{\chi_{\mathbf{n}}(\theta)} k_{\mathcal{D}^{(\mathbf{n})}}(t,[g],[h]), \\
& k_{\mathcal{D}^{(\mathbf{n})}}(t,[g],[h] * \theta)=k_{\mathcal{D}^{(\mathbf{n})}}(t,[g],[h * \theta])=\chi_{\mathbf{n}}(\theta) k_{\mathcal{D}^{(\mathbf{n})}}(t,[g],[h]),
\end{aligned}
$$

where $\theta \in \mathbb{A}$. Let $\mathbb{M}=\left\{\mu_{i}\right\}$ be a set of complete representatives of the coset space $\Gamma /(\Gamma \cap \mathbb{A})$, then the trace of the heat operator $e^{-t \mathcal{D}^{(\mathbf{n})}}$ is given as follows:

Proposition 3.3 (see [4]) For each $\mathbf{n}$ in the dual lattice $[\Gamma \cap \mathbb{A}]^{*}$ and with the heat kernel $K^{\mathbb{G}}$ of the sub-Laplacian on $\mathbb{G}$ :

$$
\begin{aligned}
& \operatorname{Vol}(\mathbb{A} /(\Gamma \cap \mathbb{A})) \cdot \operatorname{tr}\left(e^{-t \mathcal{D}^{(\mathbf{n})}}\right) \\
& =\int_{\mathcal{F}_{\Gamma}}\left(\sum_{\gamma \in \Gamma} \int_{\mathcal{F}_{\Gamma \cap \mathbb{A}}} K^{\mathbb{G}}(t, g, \gamma * g * \lambda) \overline{\chi_{\mathbf{n}}(\lambda)} d \lambda\right) d g \\
& =\int_{\mathcal{F}_{\Gamma}}\left(\sum_{\mu \in \mathbb{M}} \sum_{\nu \in \Gamma \cap \mathbb{A}} \int_{\mathcal{F}_{\Gamma \cap \mathbb{A}}} k^{\mathbb{G}}\left(t, g^{-1} * \mu * g * \nu * \lambda\right) \overline{\chi_{\mathbf{n}}(\lambda)} d \lambda\right) d g \\
& =\int_{\mathcal{F}_{\Gamma}}\left(\sum_{\mu \in \mathbb{M}} \int_{\mathbb{R}^{d}} k^{\mathbb{G}}\left(t, g^{-1} * \mu * g * \lambda\right) \overline{\chi_{\mathbf{n}}(\lambda)} d \lambda\right) d g \\
& =\sum_{\mu \in \mathbb{M}} \int_{\mathcal{F}_{\Gamma}} \int_{\mathbb{R}^{d}} k^{\mathbb{G}}\left(t, g^{-1} * \mu * g * \lambda\right) \overline{\chi_{\mathbf{n}}(\lambda)} d \lambda d g .
\end{aligned}
$$

Here denotes the set of representatives of the quotient group $\Gamma \bigcap \mathbb{A} \Gamma$. Applying Theorem 2.2 we can give a more concrete expression of the formula in Proposition 3.3. For this purpose and for the sake of simplicity, we assume that the structure constants $c_{i j}^{k}$ in (2.2) are of the form

$$
c_{i j}^{k}=\frac{2 q_{i j}^{k}}{p_{0}}
$$

with a common positive integer $p_{0} \geq 1$ and integers $q_{i j}^{k}$. Then we fix a lattice $\Gamma$

$$
\Gamma:=\left\{\sum_{1 \leq i \leq N} m_{i} X_{i}+\sum_{1 \leq k \leq d} \frac{\ell_{k}}{p_{0}} Z_{k} \mid m_{i}, \ell_{k} \in \mathbb{Z}\right\},
$$

and we choose the set $\mathbb{M}=\left\{\mu=\sum_{1 \leq i \leq N} m_{i} X_{i} \mid m_{i} \in \mathbb{Z}\right\}$ of complete representatives of the quotient group $(\Gamma \cap \mathbb{A}) \backslash \Gamma=\Gamma /(\Gamma \cap \mathbb{A})$. For each fixed

$$
\mathbf{n}=p_{0} \sum_{k=1}^{d} n_{k} Z_{k} \in[\Gamma \cap \mathbb{A}]^{*}
$$

where $n_{k} \in \mathbb{Z}$ we have

$$
\begin{aligned}
& \operatorname{Vol}(\mathbb{A} /(\Gamma \cap \mathbb{A})) \cdot \operatorname{tr}\left(e^{-t \mathcal{D}^{(\mathbf{n})}}\right) \\
& \quad=\frac{1}{(2 \pi t)^{N / 2+d}} \int_{\mathcal{F}_{\Gamma}} \sum_{\mu \in \mathbb{M}} \int_{\mathbb{A}} \int_{\mathbb{R}^{d}} e^{-\sqrt{-1} \frac{\{[\mu, x]+\lambda, \tau\rangle}{t}} \cdot \varphi_{t}(\tau, \mu) d \tau \overline{\chi_{\mathbf{n}}(\lambda)} d \lambda d x d z=(*),
\end{aligned}
$$


where the function $\varphi_{t}(\tau, \mu)$ in the integrand is given by:

$$
\varphi_{t}(\tau, \mu)=\exp \left\{-\frac{1}{2 t}\langle\Omega(\sqrt{-1} \tau) \operatorname{coth} \Omega(\sqrt{-1} \tau) \cdot \mu, \mu\rangle\right\} W(\tau) .
$$

In the following we write $\widehat{\varphi}_{t}(\tau, \mu)$ for the Fourier transform of $\varphi_{t}$ with respect to the $\tau$ variable. Then

$$
\begin{aligned}
(*) & =\frac{1}{t^{N / 2+d} \cdot(2 \pi)^{(N+d) / 2}} \int_{\mathcal{F}_{\Gamma}} \sum_{\mu \in \mathbb{M}} \int_{\mathbb{A}} \widehat{\varphi}_{t}\left(\frac{[\mu, x]+\lambda}{t}, \mu\right) \cdot e^{-2 \pi \sqrt{-1}\langle\mathbf{n}, \lambda\rangle} d \lambda d x d z \\
& =\frac{1}{t^{N / 2+d} \cdot(2 \pi)^{(N+d) / 2}} \int_{\mathcal{F}_{\Gamma}} \sum_{\mu \in \mathbb{M}} \int_{\mathbb{A}} \widehat{\varphi}_{t}(u, \mu) \cdot e^{-2 \pi \sqrt{-1}\langle\mathbf{n}, t u+[x, \mu]\rangle} t^{d} d u d x d z, \\
& =\frac{1}{(2 \pi t)^{N / 2}} \cdot p_{0}{ }^{d} \cdot \sum_{\mu \in \mathbb{M}} \varphi_{t}(-2 \pi t \mathbf{n}, \mu) \cdot \underbrace{\int_{[0,1] \times \cdots \times[0,1]}}_{N \text { times }} e^{-2 \pi \sqrt{-1}\langle\mathbf{n},[x, \mu]\rangle} d x .
\end{aligned}
$$

With a suitable set of linear independent vectors $a_{1}(\mathbf{n}), \ldots, a_{b(\mathbf{n})}(\mathbf{n})$ in $\Gamma$ the solution space $\mathbb{M}(\mathbf{n})=\{\mu \in \mathbb{M} \mid \Omega(\mathbf{n})(\mu)=0\}$ can be written as

$$
\mathbb{M}(\mathbf{n})=\left\{\mu=\sum_{i=1}^{b(\mathbf{n})} m_{i} a_{i}(\mathbf{n}), \mid m_{i} \in \mathbb{Z}\right\} .
$$

Here $b(\mathbf{n}) \leq N$ and $b(\mathbf{n})=N$ if and only if $\mathbf{n}=0$. Hence

Theorem 3.4 For each $\mathbf{n}$ in the dual lattice $[\Gamma \cap \mathbb{A}]^{*}$ and with the above notation:

$$
\begin{aligned}
\operatorname{tr}\left(e^{-t \mathcal{D}^{(\mathbf{n})}}\right) & =\frac{1}{(2 \pi t)^{N / 2}} \sum_{\mu \in \mathbb{M}(\mathbf{n})} e^{-\frac{\langle\Omega(2 \pi t \sqrt{-1} \mathbf{n}) \operatorname{coth} \Omega(2 \pi \sqrt{-1} t \mathbf{n}) \mu, \mu}{2 t}} \sqrt{\operatorname{det} \frac{\Omega(2 \pi \sqrt{-1} t \mathbf{n})}{\sinh \Omega(2 \pi \sqrt{-1} t \mathbf{n})}} \\
& =\frac{1}{(2 \pi t)^{N / 2}} \sum_{\mu \in \mathbb{M}(\mathbf{n})} e^{-\frac{<\mu, \mu>}{2 t}} \sqrt{\operatorname{det} \frac{\Omega(2 \pi \sqrt{-1} t \mathbf{n})}{\sinh \Omega(2 \pi \sqrt{-1} t \mathbf{n})} .}
\end{aligned}
$$

In particular, it holds:

$$
\operatorname{tr}\left(e^{-\Delta_{\text {sub }}^{\Gamma \backslash \mathbb{G}}}\right)=\frac{1}{(2 \pi t)^{N / 2}} \sum_{\mathbf{n} \in[\Gamma \cap \mathbb{A}]^{*}} \sum_{\mu \in \mathbb{M}(\mathbf{n})} e^{-\frac{<\mu, \mu>}{2 t}} \sqrt{\operatorname{det} \frac{\Omega(2 \pi \sqrt{-1} t \mathbf{n})}{\sinh \Omega(2 \pi \sqrt{-1} t \mathbf{n})}} .
$$

Proof It suffices to show the second equation in (3.4). Note that the defining equation $\Omega(\mathbf{n})(\mu)=0$ for $\mu \in \mathbb{M}(\mathbf{n})$ implies:

$$
\langle\Omega(2 \pi t \sqrt{-1} \mathbf{n}) \operatorname{coth} \Omega(2 \pi \sqrt{-1} t \mathbf{n})(\mu), \mu\rangle=\langle\mu, \mu\rangle=\sum m_{i} m_{j}\left\langle a_{i}(\mathbf{n}), a_{j}(\mathbf{n})\right\rangle .
$$

The last statement follows from (3.2) and (3.4).

Corollary 3.5 For $\mathbf{n},-\mathbf{n} \in[\Gamma \cap \mathbb{A}]^{*}$ the traces $\operatorname{tr}\left(e^{-t \mathcal{D}^{(\mathbf{n})}}\right)$ and $\mathbf{t r}\left(e^{-t \mathcal{D}^{(-\mathbf{n})}}\right)$ coincide.

\section{Pseudo $\boldsymbol{H}$-type algebras and groups}

For the rest of the paper we consider a specific subclass of all 2-step nilpotent Lie groups, the so called pseudo H-type groups. These are generalizations of Heisenberg type groups 
in $[23,24]$ and have been first introduced in [10]. An extensive analysis of the structure and classification of pseudo $H$-type groups and their algebras can be found in the recent papers [13-15]. For completeness we recall the relevant definitions:

We write $\mathbb{R}^{r, s}$ for the Euclidean space $\mathbb{R}^{r+s}$ equipped with the non-degenerate scalar product

$$
\langle x, y\rangle_{r, s}:=\sum_{i=1}^{r} x_{i} y_{i}-\sum_{j=1}^{s} x_{r+j} y_{r+j} .
$$

Consider the quadratic form $q_{r, s}(x)=\langle x, x\rangle_{r, s}$ and let $C \ell_{r, s}$ denote the Clifford algebra generated by $\left(\mathbb{R}^{r, s}, q_{r, s}\right)$ [25]. We call a $C \ell_{r, s}$-module $V$ admissible, if there is a nondegenerate bilinear form (= scalar product) $\langle\bullet, \bullet\rangle_{V}$ on $V$ satisfying the following conditions:

(a) There is a Clifford module action $J: C \ell_{r, s} \times V \rightarrow V:(z, X) \mapsto J_{z} X$, i.e.

$$
J_{z} J_{z^{\prime}}+J_{z^{\prime}} J_{z}=-2\left\langle z, z^{\prime}\right\rangle_{r, s} I \text { for all } z, z^{\prime} \in \mathbb{R}^{r, s} .
$$

(b) For all $z \in \mathbb{R}^{r, s}$ the map $J_{z}$ is skew-symmetric on $V$ with respect to $\langle\bullet, \bullet\rangle_{V}$, i.e.

$$
\left\langle J_{z} X, Y\right\rangle_{V}+\left\langle X, J_{z} Y\right\rangle_{V}=0 \text { for all } X, Y \in V .
$$

Moreover, from (a) and (b) one concludes:

$$
\left\langle J_{z} X, J_{z} Y\right\rangle_{V}=\langle z, z\rangle_{r, s}\langle X, Y\rangle_{V} \text { where } X, Y \in V, \quad z \in \mathbb{R}^{r, s} .
$$

We write $\left\{J, V,\langle\bullet, \bullet\rangle_{V}\right\}$ for an admissible module of the Clifford algebra $C \ell_{r, s}$ with the module action $J=J_{z}$ and the scalar product $\langle\bullet, \bullet\rangle_{V}$.

Remark 4.1 The existence of an admissible $C \ell_{r, s}$-module $V$ has been shown in [10]. If $s \neq 0$ then an admissible module $V$ needs not to be irreducible. More precisely, five cases are possible which all are present in the classification. If $C \ell_{r, s}$ has, up to equivalence, only one irreducible representation $(J, V)$, then either $V$ or the sum $V \oplus V$ is admissible. In the case where $C \ell_{r, s}$ has two non-equivalent irreducible representations $\left(J^{(i)}, V_{i}\right), i=1,2$, then either $V_{i}$ for $i=1,2$ both are admissible, or only $V_{1} \oplus V_{2}$ is admissible, or $V_{1} \oplus V_{1}$ and $V_{2} \oplus V_{2}$ simultaneously are admissible. These cases are complementary to each other (cf. [10,13-15]).

In the case $s=0$ the situation is simpler. Every irreducible module $V$ is admissible with respect to an inner product (i.e. $\langle\bullet, \bullet\rangle_{V}$ is positive definite). Originally such cases have been defined and studied by Kaplan in [23].

In the following, we call a vector $X \in V$ positive (resp. negative) if the scalar product $\langle X, X\rangle_{V}$ is positive (resp. negative) and null vector if $\langle X, X\rangle_{V}=0$. A similar notation is used for vectors $Z \in \mathbb{R}^{r, s}$. If $s>0$, then an admissible module $V$ with scalar product $\langle\bullet, \bullet\rangle_{V}$ has positive and negative subspaces of the same dimension $N$ with respect to the above scalar product $\langle\bullet, \bullet\rangle_{V}$, cf. [10]. In particular, $\operatorname{dim} V=2 N$ is even.

Moreover, $V$ decomposes into the orthogonal sum of minimal dimensional admissible modules. In fact, since the scalar product restricted to such an invariant subspace is nondegenerate the orthogonal complement is also an admissible module.

Definition 4.2 Let $\left\{J, V,\langle\bullet, \bullet\rangle_{V}\right\}$ be an admissible $C \ell_{r, s}$-module.

(1) The 2-step nilpotent Lie algebra $V \oplus_{\perp} \mathbb{R}^{r, s}$ with center $\mathbb{R}^{r, s}$ and Lie brackets defined via the relation

$$
\left\langle J_{z}(X), Y\right\rangle_{V}=\langle z,[X, Y]\rangle_{r, s}, \quad z \in \mathbb{R}^{r, s}, \text { and } X, Y \in V,
$$


will be denoted by $\mathcal{N}_{r, s}(V)$. We write $\mathbb{G}_{r, s}(V)$ for the corresponding simply connected Lie group and call it a pseudo $H$-type group, cf. [10,13].

(2) If $V$ is of minimal dimension among all admissible modules, then we call $V$ minimal admissible and we shortly write $\mathcal{N}_{r, s}:=\mathcal{N}_{r, s}(V)$ and $\mathbb{G}_{r, s}:=\mathbb{G}_{r, s}(V)$.

Remark 4.3 Note that minimal admissible modules are cyclic and the nilpotent Lie algebra $\mathcal{N}_{r, s}$ is unique up to isomorphisms, even if the Clifford algebra $C \ell_{r, s}$ admits two nonequivalent irreducible representations (cf. [13]).

We fix an orthonormal basis $\left\{Z_{k}\right\}_{k=1}^{r+s}$ in $\mathbb{R}^{r, s}$, i.e. we assume that:

$$
\begin{aligned}
& \left\langle Z_{i}, Z_{i}\right\rangle_{r, s}=1(i=1, \ldots, r),\left\langle Z_{r+j}, Z_{r+j}\right\rangle_{r, s}=-1(j=1, \ldots, s), \text { and } \\
& \left\langle Z_{i}, Z_{j}\right\rangle_{r, s}=0(i \neq j) .
\end{aligned}
$$

Let $\left\{J, V,\langle\bullet, \bullet\rangle_{V}\right\}$ be an admissible $C \ell_{r, s}$-module.

Theorem 4.4 (cf. $[11,13]$ ) Assume that $s>0$. Then there exists an orthonormal basis $\left\{X_{i}\right\}_{i=1}^{2 N}$ of $V$ such that

(1) $\left\langle X_{i}, X_{i}\right\rangle_{V}=1(i=1 \ldots, N),\left\langle X_{i}, X_{i}\right\rangle_{V}=-1(i=N+1, \ldots, 2 N)$ and

$$
\left\langle X_{i}, X_{j}\right\rangle_{V}=0 \text { for } i \neq j
$$

(2) For each $k$, the operator $J_{Z_{k}}$ maps $X_{i}$ to some $X_{j}$ or $-X_{j}$ with $j \neq i$.

Definition 4.5 We call a basis $\left\{X_{i}, Z_{j}\right\}$ satisfying the properties in Theorem 4.4 an integral basis of the algebra $\mathcal{N}_{r, s}(V)$.

Remark 4.6 An interesting problem, which we will postpone to a future work, consists in a classification of integral bases up to isomorphisms. Consider an orthonormal basis $\left\{Z_{k}\right\}_{k=1}^{r+s}$ of $\mathbb{R}^{r, s}$ in the above sense. If $V$ is a minimal admissible $\mathrm{C} \ell_{r, s}$-module, then we can define a finite subgroup $G$ in $\mathrm{GL}(V)$ generated by $\left\{J_{Z_{k}}: k=1, \ldots, r+s\right\}$. Consider the commutative subgroup:

$$
\mathbb{S}:=\left\{A \in \mathbb{G}: A^{2}=\mathrm{Id}, A=J_{Z_{i_{1}}} \ldots J_{Z_{i_{r}}}>0, A \neq-\mathrm{Id}\right\} \subset G .
$$

By " $A>0$ " we mean that $A$ maps positive (resp. negative) vectors in $V$ to positive (resp. negative) vectors. Such groups are partially ordered with respect to the inclusion and we assume that $\mathbb{S}$ is a maximal element. Further, we assume that $v \in V$ is a common eigenvector of elements in $\mathbb{S}$. Necessarily, $v$ is not a null vector, i.e. $\langle v, v\rangle_{V} \neq 0$. Consider

$$
\left\{ \pm X_{i}\right\}=\{A v: A \in G\} .
$$

We conjecture that a suitable choice of the common eigenvector $v$ leads to an integral basis $\left\{X_{i}, Z_{\ell}\right\}$ of the pseudo $H$-type Lie algebra $\mathcal{N}_{r, s}(V)$.

Conversely, let $\left\{X_{i}, Z_{j}\right\}$ be an integral basis and put $\pm \mathcal{B}:=\left\{ \pm X_{\ell}: \ell=1, \ldots, m=\right.$ $\operatorname{dim} V$ \}. Then each $J_{Z_{i}}$ defines a bijective map

$$
J_{Z_{i}}: \pm \mathcal{B} \rightarrow \pm \mathcal{B}
$$

and elements in the group $G$ act on $\pm \mathcal{B}$. We obtain a subgroup $\mathbb{S}$ as above from this basis by defining

$$
\left\{A \in \mathbb{G}: A\left(X_{1}\right)=X_{1}\right\}=: \mathbb{S} \subset G .
$$

We conjecture that every maximal subgroup $\mathbb{S}$ defines an integral basis. A classification of integral bases up to isomorphisms is left as an interesting problem, which we postpone to a future study. 
From now on we assume that $\left\{X_{i}, Z_{k}\right\}$ is an integral basis of $\mathcal{N}_{r, s}(V)$.

Corollary 4.7 If there exists $i \in\{1, \ldots, 2 N\}$ such that $J_{Z_{k}}\left(X_{i}\right)= \pm J_{Z_{\ell}}\left(X_{i}\right)$, then $k=\ell$. Hence any basis vector $X_{i}$ is mapped to some $X_{j}$ or $-X_{j}$ by at most one operator $J_{Z_{k}}$.

Proof If $k \leq r$ then $J_{Z_{k}}$ maps positive to positive and negative to negative elements. Similarly, if $k>r$, then $J_{Z_{k}}$ maps positive to negative and negative to positive elements. Therefore, under the above assumption only the cases $k, \ell \leq r$ or $k, \ell>r$ are possible.

Let us assume $k \neq \ell$ such that $\pm X_{i}=J_{Z_{k}} J_{Z_{\ell}}\left(X_{i}\right)$. By the previous remark we have

$$
J_{Z_{k}} J_{Z_{\ell}} \circ J_{Z_{k}} J_{Z_{\ell}}=-J_{Z_{k}} \circ J_{Z_{\ell}}^{2} \circ J_{Z_{k}}=-\left\langle Z_{k}, Z_{k}\right\rangle_{r, s}\left\langle Z_{\ell}, Z_{\ell}\right\rangle_{r, s}=-I .
$$

This equation contradicts the existence of the eigenvalue 1 or -1 of $J_{Z_{k}} J_{Z_{\ell}}$.

Corollary 4.8 If we put $\left[X_{i}, X_{j}\right]=\sum c_{i j}^{k} Z_{k}$, then $c_{i j}^{k}$ can be non-zero for at most one $k$. If $c_{i j}^{k}$ is non-zero then it equals \pm 1 .

Proof The statement follows from Corollary 4.7 and

$$
\left\langle J_{Z_{\ell}} X_{i}, X_{j}\right\rangle_{V}=\left\langle Z_{\ell},\left[X_{i}, X_{j}\right]\right\rangle_{r, s}= \begin{cases}c_{i j}^{\ell} & \text { if } \ell \leq r \\ -c_{i j}^{\ell} & \text { if } \ell>r .\end{cases}
$$

Definition 4.9 From an integral basis $\left\{X_{i}, Z_{k}\right\}$ of $\mathcal{N}_{r, s}(V)$ we define a lattice in the pseudo $H$-type group $\mathbb{G}_{r, s}(V)$ by

$$
\Gamma_{r, s}(V):=\left\{\sum_{m_{i} \in \mathbb{Z}} m_{i} X_{i}+\frac{1}{2} \sum_{k_{j} \in \mathbb{Z}} k_{j} Z_{j}\right\} .
$$

In the following we call $\Gamma_{r, s}(V)$ a standard integral lattice in $\mathcal{N}_{r, s}(V)$. If $\mathcal{N}_{r, s}$ is constructed from a minimal admissible module $V$ (cf. Definition 4.2), then we write $\Gamma_{r, s}:=\Gamma_{r, s}(V)$.

Remark 4.10 A standard integral lattice is not unique. A complete classification will be subject of another work. For particular cases the construction of $\Gamma_{r, s}$ is found in [13].

In the following two sections we consider the sub-Laplacian

$$
\Delta_{\mathrm{sub}}^{\mathbb{G}_{r, s}(V)}=-\frac{1}{2} \sum_{i=1}^{2 N} \tilde{X}_{i}^{2}
$$

on $\mathbb{G}_{r, s}(V)$, where $\left\{X_{i}: i=1, \ldots, 2 N\right\}$ is the basis of the module $V$ in the definition of the standard integral lattice $\Gamma_{r, s}(V)$. We determine the heat trace of the sub-Laplacian

$$
\Delta_{\mathrm{sub}}^{\Gamma_{r, s}(V) \backslash \mathbb{G}_{r, s}(V)}
$$

descended from (4.5) to the nilmanifold $\Gamma_{r, s}(V) \backslash \mathbb{G}_{r, s}(V)$. Based on the sub-ellipticity of (4.6) it is known that the spectrum of the sub-Laplacian only consists of eigenvalues with finite multiplicities. In principle our trace formula in Theorem 3.4 can be used to obtain the spectrum of (4.6). However, we will not calculate the eigenvalues and multiplicities explicitly since a comparison of heat traces is sufficient to decide isospectrality. 


\section{The structure constants of pseudo $H$-type groups}

In the case of pseudo $H$-type groups we calculate the characteristic polynomial of the matrix $\Omega(z)$ in (2.3) in the case where $s>0$ in Definition 4.2 of the pseudo $H$-type group $\mathbb{G}_{r, s}(V)$. Recall that this matrix is an essential ingredient for the integral expression of the heat kernel in Theorem 2.2.

Throughout this section we assume that $s>0$ so that we can use the integral basis in Theorem 4.4. Let us start by decomposing the Clifford module $V=V_{+} \oplus_{\perp} V_{-}$into a positive and a negative subspace $V_{+}:=\left[X_{i}: i=1, \ldots, N\right]$ and $V_{-}=\left[X_{N+j}: j=1, \ldots, N\right]$, where $\left\{X_{i}: i=1, \ldots, 2 N\right\}$ is part of a standard integral basis $\left\{X_{i}, Z_{j}\right\}$ of $\mathcal{N}_{r, s}(V)$. Then the Clifford module action

$$
J_{z}\left(X_{i}\right)=\sum_{j} c_{i j}(z) X_{j}
$$

with

$$
z=\sum_{i=1}^{r} \mu_{i} Z_{i}+\sum_{j=1}^{s} v_{j} Z_{r+j} \cong(\mu, v)^{T} \in \mathbb{R}^{r, s}
$$

can be written in form of a matrix with respect to the basis $\left\{X_{i}\right\}$ of $V$ :

$$
J_{z}=\left(\begin{array}{ll}
A & B \\
C & D
\end{array}\right): \begin{array}{ccc}
V_{+} & V_{+} \\
\bigoplus_{\perp} & \longrightarrow & \oplus_{\perp} . \\
V_{-} & V_{-}
\end{array}
$$

By (4.3) the map $J_{z}$ leaves $V_{ \pm}$invariant whenever $z$ is positive in $\mathbb{R}^{r, s}$. If $z \in \mathbb{R}^{r, s}$ is negative then $J_{z}$ maps $V_{+}$to $V_{-}$and vice versa. It follows that the component matrices $A, B, C, D$ are of the forms $A=A(\mu), B=B(\nu), C=C(\nu)$ and $D=D(\mu)$. Due to the admissibility condition (4.2) of the Clifford action on the module $V$ we have

$$
A^{T}(\mu)=-A(\mu), \quad B^{T}(v)=C(\nu) \text { and } D^{T}(\mu)=-D(\mu) .
$$

Here $A^{T}(\mu)$ denotes the transposed matrix of $A(\mu) \in \mathbb{R}(N)$. Moreover, the identity $J_{z}^{2}=-\langle z, z\rangle_{r, s}$ yields additional relations between the component matrices $A, B, C$ and $D$ which are collected in the next lemma.

Lemma 5.1 With the notion $\|\mu\|^{2}:=\sum_{i=1}^{r} \mu_{i}^{2}$ and $\|v\|^{2}:=\sum_{j=1}^{s} v_{j}^{2}$ we have:

(a) $A(\mu)^{2}+B(v) C(v)=-\langle z, z\rangle_{r, s}=-\left(\|\mu\|^{2}-\|v\|^{2}\right)$,

(b) $A(\mu) B(v)+B(v) D(\mu)=0$ and $C(v) A(\mu)+D(\mu) C(v)=0$,

(c) $C(v) B(v)+D(\mu)^{2}=-\langle z, z\rangle_{r, s}$.

In particular, it follows $A(\mu)^{2}=-\|\mu\|^{2}$ and $D(\mu)^{2}=-\|\mu\|^{2}$.

Let $L$ be a linear map on $V \cong \mathbb{R}^{2 N}$. The same notation is used for its matrix representation with respect to the basis $\left\{X_{i}\right\}$. Let $\langle\cdot, \cdot\rangle$ denote the Euclidean inner product on $\mathbb{R}^{2 N}$ and fix $x, y \in V$. We calculate the matrix representation of the transpose $L^{*}$ of $L$ with respect to the scalar product $\langle\cdot, \cdot\rangle_{V}$. Consider the matrix

$$
\tau:=\left(\begin{array}{cc}
I & 0 \\
0 & -I
\end{array}\right) \in \mathbb{R}(2 N) \text { where } I=\text { identity } \in \mathbb{R}(N) .
$$

Then we have

$$
\langle L x, y\rangle_{V}=\langle L x, \tau y\rangle=\left\langle x, L^{T} \tau y\right\rangle=\left\langle x, \tau L^{T} \tau y\right\rangle_{V},
$$


which implies that $L^{*}=\tau L^{T} \tau$. A direct calculation shows that the skew-symmetric matrix $\Omega(z)$ in (2.3) is related to the above matrix representation of $J_{z}$ as follows:

$$
\Omega(z)=\tau J_{z}^{T}=\left(\begin{array}{cc}
-A(\mu) & B(v) \\
-B^{T}(\nu) & D(\mu)
\end{array}\right) .
$$

Remark 5.2 If $r=0$, then $A(\mu)=D(\mu)=0$.

In order to determine the eigenvalues of the matrix $\Omega(\sqrt{-1} z)$ we employ the relation

$$
\underbrace{\left(\begin{array}{cc}
-A+\lambda & B \\
-C & D+\lambda
\end{array}\right)}_{=\Omega(z)+\lambda}\left(\begin{array}{cc}
I & -(-A+\lambda)^{-1} B \\
0 & I
\end{array}\right)=\left(\begin{array}{cc}
-A+\lambda & 0 \\
-B^{T} & B^{T}(-A+\lambda)^{-1} B+\lambda+D
\end{array}\right) .
$$

Hence we have

$$
\operatorname{det}(\Omega(z)+\lambda)=\operatorname{det}(-A+\lambda) \operatorname{det}\left(B^{T}(-A+\lambda)^{-1} B+\lambda+D\right) .
$$

According to Lemma 5.1 one has $B^{T} B=\|v\|^{2}=B B^{T}$ and $B^{T} A B+\|v\|^{2} D=0$, showing that $B^{T}(-A+\lambda) B=\|v\|^{2}(\lambda+D)$. Together with the skew-symmetry of $\Omega(z)$ :

$$
\begin{aligned}
& \operatorname{det}(\Omega(z)+\lambda)=\operatorname{det}(\Omega(z)-\lambda) \\
& =\operatorname{det}(-A+\lambda) \operatorname{det}\left(B^{T}(-A+\lambda)^{-1} B+\frac{1}{\|v\|^{2}} B^{T}(-A+\lambda) B\right) \\
& =\operatorname{det}(-A+\lambda) \operatorname{det}\left(B^{T}\left[(-A+\lambda)^{-1}+\frac{1}{\|v\|^{2}}(-A+\lambda)\right] B\right) \\
& =\operatorname{det}\left(\|v\|^{2}-\|\mu\|^{2}+\lambda^{2}-2 \lambda A\right) .
\end{aligned}
$$

Therefore:

$$
\begin{gathered}
\operatorname{det}(\Omega(z)+\lambda)^{2}=\operatorname{det}(\Omega(z)+\lambda) \operatorname{det}(\Omega(z)-\lambda) \\
=\operatorname{det}\left(\left(\|v\|^{2}-\|\mu\|^{2}+\lambda^{2}\right)^{2}+4 \lambda^{2}\|\mu\|^{2}\right) .
\end{gathered}
$$

Proposition 5.3 With $s>0$ and $z \in \mathbb{R}^{r, s}$ we have:

$$
\begin{aligned}
\operatorname{det}(\Omega(z)+\lambda)^{2} & =\left(\left(\lambda^{2}+\|\mu\|^{2}+\|v\|^{2}\right)^{2}-4\|\mu\|^{2}\|v\|^{2}\right)^{N} \\
& =\left[\left(\lambda^{2}+(\|\mu\|+\|v\|)^{2}\right)\left(\lambda^{2}+(\|\mu\|-\|v\|)^{2}\right)\right]^{N} .
\end{aligned}
$$

By replacing $z$ with $\sqrt{-1} z, \mu$ with $\sqrt{-1} \mu$ and $v$ with $\sqrt{-1} v$ we have:

Corollary 5.4 The eigenvalues $\lambda$ of the matrix $\Omega(\sqrt{-1} z)$ are $\lambda= \pm(\|\mu\| \pm\|v\|)$.

If $z \neq 0$ then the matrix $\Omega(z)$ has the eigenvalue zero only when $\|\mu\|=\|v\|$. In this case the matrices $B(v)$ and $D(\mu)$ are non-singular so that the dimension of the solution space $\Omega(z) \cdot x=0$ is $N$ (= half the dimension of $V$ ).

Proposition 5.5 Assume that $z \neq 0$ and $\|\mu\|=\|v\|$. The kernel of $\Omega(z)$ is given by

$$
\operatorname{Ker} \Omega(z)=\left\{\left(\begin{array}{c}
B(\nu) x \\
-D(\mu) x
\end{array}\right) \mid x=\left(x_{1}, \ldots, x_{N}\right)^{T} \in \mathbb{R}^{N}\right\} .
$$


Finally we determine the dimension of the eigenspaces corresponding to the above eigenvalues $\pm(\|\mu\| \pm\|v\|)$.

Proposition 5.6 Let $z \neq 0$. Then the dimensions of the eigenspaces $E_{\lambda}$ of $\Omega(\sqrt{-1} z)$ with respect to the eigenvalue $\lambda= \pm(\|\mu\| \pm\|v\|)$ are given as follows:

(i) If neither $\mu$ nor $v$ is zero, then $\operatorname{dim} E_{\lambda}=N / 2$ and therefore $\frac{\operatorname{dim} V}{2}=N$ is even.

(ii) If $\mu=0$ and $v \neq 0$, then $\operatorname{dim} E_{\|v\|}=N=\operatorname{dim} E_{-\|v\|}$.

(iii) If $\mu \neq 0$ and $v=0$, then $\operatorname{dim} E_{\|\mu\|}=N=\operatorname{dim} E_{-\|\mu\|}$.

Proof (i): Let $(x, y)^{T} \in \mathbb{R}^{N} \times \mathbb{R}^{N} \cong \mathbb{R}^{2 N}$ be an eigenvector of the matrix $\Omega(\sqrt{-1} z)$ with respect to the eigenvalue $\lambda=\|\mu\|+\|v\|$, where $\mu, v \neq 0$, then

$$
\Omega(\sqrt{-1} z)\left(\begin{array}{l}
x \\
y
\end{array}\right)=\left(\begin{array}{ll}
-A(\sqrt{-1} \mu) & B(\sqrt{-1} v) \\
-C(\sqrt{-1} v) & D(\sqrt{-1} \mu)
\end{array}\right)\left(\begin{array}{l}
x \\
y
\end{array}\right)=(\|\mu\|+\|v\|)\left(\begin{array}{l}
x \\
y
\end{array}\right) .
$$

Multiplying the first equation by $A(\sqrt{-1} \mu)$ and the second equation by $B(\sqrt{-1} \nu)$ gives

$$
\begin{aligned}
& A(\sqrt{-1} \mu)(-A(\sqrt{-1} \mu) x+B(\sqrt{-1} v) y)=(\|\mu\|+\|v\|) A(\sqrt{-1} \mu) x \\
& B(\sqrt{-1} v)(-C(\sqrt{-1} v) x+D(\sqrt{-1} \mu) y)=(\|\mu\|+\|v\|) B(\sqrt{-1} v) y .
\end{aligned}
$$

On the left hand side we use Lemma 5.1 and deduce the following two equations

$$
\begin{aligned}
-\|\mu\|^{2} x+A(\sqrt{-1} \mu) B(\sqrt{-1} \nu) y & =(\|\mu\|+\|\nu\|) A(\sqrt{-1} \mu) x \\
\|v\|^{2} x+B(\sqrt{-1} \nu) D(\sqrt{-1} \mu) y & =(\|\mu\|+\|\nu\|) B(\sqrt{-1} \nu) y .
\end{aligned}
$$

Adding these identities and using Lemma 5.1, (ii) gives

$$
(\|v\|-\|\mu\|) x=A(\sqrt{-1} \mu) x+B(\sqrt{-1} v) y .
$$

Together with the Eq. (5.2) we find that $B(\sqrt{-1} v) y=\|v\| x$. This shows that

$$
A(\sqrt{-1} \mu) x=-\|\mu\| x \text {. }
$$

Since $B(v)$ is non-singular for $v \neq 0$ the vector $y$ is uniquely determined by $x$. Conversely, the eigenvector $x$ of the matrix $A(\sqrt{-1} \mu)$ with the eigenvalue $-\|\mu\| \neq 0$, determines the eigenvector of the matrix $\Omega(\sqrt{-1} z)$ by putting $y=\|v\| B(\sqrt{-1} v)^{-1} x$.

Since $A(\mu)$ is skew-symmetric for any real vector $\mu$ and $A(\mu)^{2}=-\|\mu\|^{2}$, the dimension of the eigenspace of the matrix $A(\sqrt{-1} \mu)$ with respect to the eigenvalue $\|\mu\|$ is half the size of the matrix $A$, i.e. it equals $\frac{N}{2}$. The remaining eigenvalues can be treated similarly and therefore (i) follows.

(ii): Under the assumption of (ii) and by applying the relations in Lemma 5.1 we have $A=D=0$ and $B(v) C(v)=\|v\|^{2}=C(v) B(v)$. Let $(x, y)^{T} \in \mathbb{R}^{N} \times \mathbb{R}^{N} \cong \mathbb{R}^{2 N}$ be an eigenvector of $\Omega(\sqrt{-1} z)$ with eigenvalue $\lambda=\|v\|$. Then the equation

$$
\Omega(\sqrt{-1} z)\left(\begin{array}{l}
x \\
y
\end{array}\right)=\left(\begin{array}{cc}
0 & B(\sqrt{-1} v) \\
\|v\|^{2} B^{-1}(\sqrt{-1} v) & 0
\end{array}\right)\left(\begin{array}{l}
x \\
y
\end{array}\right)=\|v\|\left(\begin{array}{l}
x \\
y
\end{array}\right)
$$

is equivalent to $B(\sqrt{-1} v) y=\|v\| x$, which can be uniquely solved for any given $x \in \mathbb{R}^{N}$. The case $\lambda=-\|v\|$ is treated in the same way and (ii) follows.

(iii): If $\mu \neq 0$ and $v=0$, then $C=B=0$ and with $\lambda=\|\mu\|$ we have the equation

$$
\Omega(\sqrt{-1} z)\left(\begin{array}{l}
x \\
y
\end{array}\right)=\left(\begin{array}{cc}
-A(\sqrt{-1} \mu) & 0 \\
0 & D(\sqrt{-1} \mu)
\end{array}\right)\left(\begin{array}{l}
x \\
y
\end{array}\right)=\|\mu\|\left(\begin{array}{l}
x \\
y
\end{array}\right) .
$$


The matrix $\Omega(\sqrt{-1} z)$ is Hermitian and therefore can be diagonalized. From the expression of det $(\Omega(z)+\lambda)^{2}$, we deduce that the eigenspaces have dimension $N$. The case $\lambda=-\|\mu\|$ can be treated similarly.

Corollary 5.7 Let $s>0$, then the characteristic polynomial of $\Omega(\sqrt{-1} z)$ is given by:

$$
\operatorname{det}(\Omega(\sqrt{-1} z)+\lambda)=\left(\lambda^{2}-(\|\mu\|+\|v\|)^{2}\right)^{N / 2}\left(\lambda^{2}-(\|\mu\|-\|v\|)^{2}\right)^{N / 2} .
$$

Remark 5.8 In the case $s=0$ we can find an admissible module $V$ with respect to an inner product ( $=$ positive definite scalar product) and so we obtain:

$$
\Omega(z)=J_{z} \text { and } \Omega(z)^{2}=J_{z}^{2}=-\|z\|^{2} I .
$$

Therefore, the statement of Corollary 5.7 remains valid even in this case:

$$
\operatorname{det}(\Omega(\sqrt{-1} z)+\lambda)=\left(\lambda^{2}-\|z\|^{2}\right)^{N} .
$$

\section{Spectrum of the sub-Laplacian on pseudo $H$-type nilmanifolds}

Let $\left\{J, V,\langle\bullet, \bullet\rangle_{V}\right\}$ be an admissible module of the Clifford algebra $C \ell_{r, s}$. Based on the results of the previous sections we derive an explicit expression for the heat trace of the sub-Laplacian $\Delta_{\text {sub }}^{\Gamma_{r, s}(V) \backslash \mathbb{G}_{r, s}(V)}$ on the nilmanifolds $\Gamma_{r, s}(V) \backslash \mathbb{G}_{r, s}(V)$ for $r>0$ and $s>0$. In fact, with a view to the decomposition (3.2), it suffices to calculate the heat trace of each component operator $\mathcal{D}^{(\mathbf{n})}$ with respect to the element $\mathbf{n}$ in the dual $\left[\mathbb{A} \cap \Gamma_{r, s}(V)\right]^{*}$ of the lattice $\mathbb{A} \cap \Gamma_{r, s}(V)$.

Recall that $\mathbb{A} \cong \mathbb{R}^{r, s}$ denotes the center of the group $\mathbb{G}_{r, s}(V)$. However, our notation will not indicate the dependence on the parameter $(r, s)$.

\subsection{Determination of the spectrum}

An element $\mathbf{n}$ in the dual lattice $\left[\Gamma_{r, s} \cap \mathbb{A}\right]^{*}$ can be expressed as

$$
\mathbf{n}=2\left(\sum_{i=1}^{r} m_{i} Z_{i}+\sum_{j=1}^{s} n_{j} Z_{r+j}\right) \quad \text { where }(m, n) \in \mathbb{Z}^{r+s}
$$

We also use the notation $\mathbf{n}=2(\mu+v)$ with $\mu+v=\left(m_{1}, \ldots, m_{r}, n_{1} \ldots, n_{s}\right) \in \mathbb{Z}^{r+s}$. Now Theorem 3.4 implies:

(1) If $\mathbf{n}=0$, then the trace of the operator $e^{-t \mathcal{D}^{(0)}}$ is given by

$$
\operatorname{tr}\left(e^{-t \mathcal{D}^{(0)}}\right)=\frac{1}{(2 \pi t)^{N}} \sum_{\ell \in \mathbb{Z}^{2 N}} e^{-\frac{\|\ell\|^{2}}{2 t}} .
$$

(2) Assume that $\mathbf{n} \in\left[\Gamma_{r, s} \cap \mathbb{A}\right]^{*}$ with

$$
\sum_{i=1}^{r} m_{i}^{2}=\sum_{j=1}^{s} n_{j}^{2}
$$


and let $d_{0}>0$ be the greatest common divisor of $(\mu, v)=\left(m_{1}, \ldots, m_{r}, n_{1}, \ldots, n_{s}\right)$. Define integers $m_{i}^{\prime}$ and $n_{i}^{\prime}$ through the equations $m_{i}=m_{i}{ }^{\prime} d_{0}$ and $n_{j}=n_{j}{ }^{\prime} d_{0}$. According to Proposition 5.5 the solution space $\mathbb{M}(\mathbf{n})=\left\{\ell \in \mathbb{Z}^{2 N} \mid \Omega(\mathbf{n}) \ell=0\right\}$ is given by:

$$
\mathbb{M}(\mathbf{n})=\left\{\left(\begin{array}{c}
B\left(v^{\prime}\right) \ell \\
-D\left(\mu^{\prime}\right) \ell
\end{array}\right) \mid \ell=\left(\ell_{1}, \ldots, \ell_{N}\right)^{T} \in \mathbb{Z}^{N}\right\}
$$

where $\left(\mu^{\prime}, v^{\prime}\right)=\left(m_{1}{ }^{\prime}, \ldots, m_{r}{ }^{\prime}, n_{1}{ }^{\prime}, \ldots, n_{s}{ }^{\prime}\right)$. Hence

$$
\operatorname{tr}\left(e^{-t \mathcal{D}^{(\mathbf{n})}}\right)=\frac{1}{(\pi t)^{N / 2}} \sum_{\ell \in \mathbb{Z}^{2 N}} e^{-\frac{\|\mu\|^{2}\|\ell\|^{2}}{d_{0}^{2} t}}\left(\frac{2\|\mu\|}{\sinh (8 \pi t\|\mu\|)}\right)^{N / 2} .
$$

(3) For $\mathbf{n}=2(\mu+v)$ with $\|\mu\| \neq\|v\|$ the matrix $\Omega(\mathbf{n})$ is non-singular. In this case the solution space $\mathbb{M}(\mathbf{n})=\{0\}$ is trivial and

$$
\operatorname{tr}\left(e^{-t \mathcal{D}^{(\mathbf{n})}}\right)=2^{N}\left(\frac{\|\mu\|^{2}-\|v\|^{2}}{\sinh \{4 \pi t(\|\mu\|+\|v\|)\} \sinh \{4 \pi t(\|\mu\|-\|v\|)\}}\right)^{N / 2} .
$$

Remark 6.1 If $s=0$, then the matrix $\Omega(\mathbf{n})$ is always non-singular for $\mathbf{n} \neq 0$. In this case we find:

$$
\operatorname{tr}\left(e^{-t \mathcal{D}^{(\mathbf{n})}}\right)=\left(\frac{\|\mathbf{n}\|}{\sinh 2 \pi t\|\mathbf{n}\|}\right)^{N} .
$$

Furthermore, from the heat trace formula (3.2) we conclude that the eigenvalues of the subLaplacian on $\Gamma_{r, 0}(V) \backslash \mathbb{G}_{r, 0}(V)$ are given by

- $\lambda_{l}=2 \pi^{2}\|l\|^{2}$ for $l \in \mathbb{Z}^{2 N}$.

- $\beta_{n, m}=4 \pi\|n\|(2 m+N)$ for $n \in \mathbb{Z}^{r} \backslash\{0\}$ and $m \in \mathbb{N}$ with multiplicity $4^{N}\|n\|^{N}\left(\begin{array}{c}m+N-1 \\ N-1\end{array}\right)$.

Since $\lambda_{l}^{2} \in \pi^{4} \mathbb{Z}$ and $\beta_{n, m}^{2} \in \pi^{2} \mathbb{Z}$, we can distinguish between these different numbers, i.e. knowing the eigenvalues, we can extract the dimension of the admissible module $V$. We conclude that if two manifolds $\Gamma_{r, 0}(V) \backslash \mathbb{G}_{r, 0}(V)$ and $\Gamma_{r^{\prime}, 0}\left(V^{\prime}\right) \backslash \mathbb{G}_{r^{\prime}, 0}\left(V^{\prime}\right)$ are isospectral with respect to the sub-Laplacian, then they have the same dimension.

Based on the dependence of the above heat traces on the parameters $\|\mu\|,\|v\|$ and $N$ we conclude:

Corollary 6.2 $\Gamma_{r, s} \backslash \mathbb{G}_{r, s}$ and $\Gamma_{s, r} \backslash \mathbb{G}_{s, r}$ are isospectral with respect to the Laplacian and the sub-Laplacian, if the dimensions of their admissible modules coincide.

The pseudo $H$-type algebra $\mathcal{N}_{r, s}$ does not depend on the chosen minimal admissible module, cf. [13]. Moreover, in the above determination we do not explicitly use the assumption that the admissible module is minimal. This implies:

Theorem 6.3 If $V$ is a sum of $k$ minimal admissible modules, then the heat trace in each of the cases, (6.1), (6.2) and (6.3) above is the $k$-th power of the corresponding heat trace for the manifold $\Gamma_{r, s} \backslash \mathbb{G}_{r, s}$. Let $U$ be an admissible module of $C \ell_{s, r}$ with $\operatorname{dim} V=\operatorname{dim} U$, then the two nilmanifolds $\Gamma_{r, s}(V) \backslash \mathbb{G}_{r, s}(V)$ and $\Gamma_{s, r}(U) \backslash \mathbb{G}_{s, r}(U)$ are isospectral. 


\section{Isospectral, but non-homeomorphic nilmanifolds}

By applying Theorem 6.3 and the classification of pseudo $H$-type Lie algebras in $[14,15]$ we detect finite families of isospectral but mutually non-homeomorphic pseudo $H$-type nilmanifolds. If the module $V$ in the construction of the pseudo $H$-type Lie algebra is minimal admissible, then pairs of non-isomorphic pseudo $H$-type Lie algebras $\mathcal{N}_{r, s} \not \mathcal{N}_{s, r}$ of the same dimension $\operatorname{dim} \mathcal{N}_{r, s}=\operatorname{dim} \mathcal{N}_{s, r}$ are known (see [14,15] or Table 7.1). By choosing integral lattices $\Gamma_{r, s}$ and $\Gamma_{s, r}$ in the corresponding Lie groups $\mathbb{G}_{r, s}$ and $\mathbb{G}_{s, r}$, respectively, we first detect pairs

$$
M_{1}:=\Gamma_{r, s} \backslash \mathbb{G}_{r, s} \quad \text { and } \quad M_{2}:=\Gamma_{s, r} \backslash \mathbb{G}_{s, r}
$$

of isospectral, non-homeomorphic manifolds. The minimal dimension of such examples arise for $(r, s)=(1,3)$ in which case $\operatorname{dim} M_{1}=\operatorname{dim} M_{2}=12$. By dropping the minimality condition on the module we can produce many more examples. More generally, for any $k \in \mathbb{N}$ we can find a family $M_{1}, \ldots, M_{k}$ of nilmanifolds such that for $i, j \in\{1, \ldots, k\}$ :

$$
M_{i} \sim_{\text {isosp }} M_{j} \text { and } M_{i} \nsim_{\text {homeo }} M_{j} .
$$

Here $\sim_{\text {isosp }}$ means $i$ sospectral with respect to the sub-Laplacian and $\nsim_{\text {homeo }}$ indicates that two manifolds are non-homeomorphic. First, we explain the method of detecting nonhomeomorphic nilmanifolds.

If there is a homeomorphism between the nilmanifolds $N_{r, s}:=\Gamma_{r, s}(V) \backslash \mathbb{G}_{r, s}(V)$ and $N_{s, r}=\Gamma_{s, r}(U) \backslash \mathbb{G}_{s, r}(U)$, then their fundamental groups

$$
\pi_{1}\left(\Gamma_{r, s}(V) \backslash \mathbb{G}_{r, s}(V)\right) \cong \Gamma_{r, s}(V) \quad \text { and } \quad \pi_{1}\left(\Gamma_{s, r}(U) \backslash \mathbb{G}_{s, r}(U)\right) \cong \Gamma_{s, r}(U)
$$

are isomorphic. We can apply the following general fact from [33]:

Proposition 7.1 Any isomorphism between lattices in simply connected nilpotent Lie groups can be extended to an isomorphism between the whole groups.

From these observations we conclude:

Corollary 7.2 If the nilmanifolds $N_{r, s}$ and $N_{s, r}$ are homeomorphic, then $\mathcal{N}_{r, s}(V)$ and $\mathcal{N}_{s, r}(U)$ have to be isomorphic as Lie algebras.

\subsection{Pairs of non-homeomorphic, isospectral nilmanifolds via minimal admissible modules}

To obtain pairs $\left(M_{1}=N_{r, s}, M_{2}=N_{s, r}\right)$ of nilmanifolds with (7.2) we determine pairs $(r, s)$ such that $\operatorname{dim} N_{r, s}=\operatorname{dim} N_{s, r}$, but $N_{r, s} ¥ N_{s, r}$, i.e. both Lie algebras are not isomorphic. The classification of pseudo $H$-type algebras in Table 1 constructed from minimal admissible modules was obtained in [14,15]. This table gives us only information about the cases $0 \leq$ $r, s \leq 8$. For the remaining cases we use the following periodicity (see also [2]):

Lemma 7.3 For $(\mu, v) \in\{(8,0),(0,8)\} \bmod 8$ and $(\mu, v)=(4,4) \bmod 4$ we have

$$
N_{r, s} \cong N_{s, r} \text { iff } N_{r+\mu, s+v} \cong N_{s+v, r+\mu} .
$$

From Corollary 7.2 and Table 1 we see that for $(r, s) \in\{(3,1),(3,2),(3,7)\}$ both nilmanifolds $N_{r, s}, N_{s, r}$ have the same dimension but they are non-homeomorphic.

Hence we obtain the following result: 
Table 1 Classification of pseudo $H$-type Lie algebras defined via minimal admissible modules

\begin{tabular}{llllllllll}
\hline 8 & $\cong$ & $\cong$ & $\cong$ & $\mathrm{h}$ & & & & \\
7 & $\mathrm{~d}$ & $\mathrm{~d}$ & $\mathrm{~d}$ & $\not$ & & & & & \\
6 & $\mathrm{~d}$ & $\cong$ & $\cong$ & $\mathrm{h}$ & $\cong$ & & & & \\
5 & $\mathrm{~d}$ & $\cong$ & $\cong$ & $\mathrm{h}$ & $\cong$ & & & & \\
4 & $\cong$ & $\mathrm{h}$ & $\mathrm{h}$ & $\mathrm{h}$ & & $\cong$ & $\cong$ & & \\
3 & $\mathrm{~d}$ & $¥$ & $¥$ & & $\mathrm{~d}$ & $\mathrm{~d}$ & $\mathrm{~d}$ & 7 & $\mathrm{~d}$ \\
2 & $\cong$ & $\mathrm{h}$ & & $\not$ & $\mathrm{d}$ & $\cong$ & $\cong$ & $\mathrm{h}$ & $\cong$ \\
1 & $\cong$ & & $\mathrm{d}$ & $¥$ & $\mathrm{~d}$ & $\cong$ & $\cong$ & $\mathrm{h}$ & $\cong$ \\
0 & & $\cong$ & $\cong$ & $\mathrm{h}$ & $\cong$ & $\mathrm{h}$ & $\mathrm{h}$ & $\mathrm{h}$ & $\cong$ \\
$s / r$ & 0 & 1 & 2 & 3 & 4 & 5 & 6 & 7 & 8 \\
\hline
\end{tabular}

Notation: If $V_{\min }^{r, s}$ denotes a minimal admissible $C \ell_{r, s}$-module, then the letter ' $\mathrm{d}$ ' $=$ double (or ' $\mathrm{h}$ ' $=$ half, respectively) at the position $(r, s)$ means that $\operatorname{dim} V_{\min }^{r, s}=2 \operatorname{dim} V_{\min }^{s, r}$ (or $\operatorname{dim} V_{\min }^{r, s}=1 / 2 \operatorname{dim} V_{\min }^{s, r}$, respectively). The symbol ' $\cong$ ' indicates that the Lie algebras $\mathcal{N}_{r, s}$ and $\mathcal{N}_{s, r}$ are isomorphic while ' $¥$ ' means that they are non-isomorphic

Corollary 7.4 The following pairs of nilmanifolds are isospectral and non-homeomorphic:

(a) $\left(N_{r, s}, N_{s, r}\right)$ for $r \equiv 3 \bmod 8$ and $s \equiv 1,2,7 \bmod 8$.

(b) $\left(N_{r+4 k, s+4 k}, N_{s+4 k, r+4 k}\right)$ for $(r, s) \in\{(3,1),(3,2),(3,7)\}$ and $k \in \mathbb{N}_{0}$.

\subsection{Finite families of non-homeomorphic, isospectral nilmanifolds}

In case the module $V$ in the construction of the Lie algebra $\mathcal{N}_{r, s}(V)$ is not minimal admissible we can use the classification result in [15, Theorem 4.1.2 and Theorem 4.1.3] to determine families $\left\{M_{1}, \ldots, M_{k}\right\}$ of a given length $k \in \mathbb{N}$ of isospectral, mutually non-homeomorphic nilmanifolds, i.e. (7.2) holds. First, we fix the pair $(r, s)$ and study the Lie algebra $\mathcal{N}_{r, s}(U)$, constructed from different admissible modules. In the general case the classification of isomorphic pseudo $H$-type Lie algebras is more subtle and to state the result we need to introduce some notation from [15]. Note that for any given minimal admissible module $\left\{J, V,\langle\bullet, \bullet\rangle_{V}\right\}$ also the module $\left\{J, V,-\langle\bullet, \bullet\rangle_{V}\right\}$ is minimal admissible. The upper index in the notation $V_{\text {min; }}^{r, s ; \pm}$ indicate that the scalar product of the two minimal admissible modules $V_{\min ; \pm}^{r, s ;+}$ and $V_{\mathrm{min} ; \pm}^{r, s ;-}$ differ by a sign.

Theorem 7.5 (Furutani, Markina, [15]) Let $r \equiv 3 \bmod 4, s \equiv 1,2,3 \bmod 4$ and $U, \widetilde{U}$ be admissible modules decomposed into the direct sums:

$$
\begin{aligned}
& U=\left(\bigoplus^{p^{+}} V_{\text {min }}^{r, s ;+}\right) \bigoplus\left(\bigoplus^{p^{-}} V_{\text {min }}^{r, s ;-}\right), \\
& \tilde{U}=\left(\tilde{p}^{\tilde{p}^{+}} V_{\text {min }}^{r, s ;+}\right) \bigoplus\left(\widetilde{p}^{-} V_{\text {min }}^{r, s ;-}\right) .
\end{aligned}
$$

Then the Lie algebras $\mathcal{N}_{r, s}(U), \mathcal{N}_{r, s}(\widetilde{U})$ are isomorphic, if and only if:

$$
\left[p^{+}=\tilde{p}^{+} \quad \text { and } \quad p^{-}=\tilde{p}^{-}\right] \text {or }\left[p^{+}=\tilde{p}^{-} \quad \text { and } \quad p^{-}=\tilde{p}^{+}\right] \text {. }
$$


Let $R \in \mathbb{N}$ be fixed. If we consider admissible modules of the form

$$
U(p, q):=\left(\bigoplus^{p} V_{\min }^{r, s ;+}\right) \bigoplus\left(\bigoplus^{q} V_{\text {min }}^{r, s ;-}\right), \quad p+q=R,
$$

then we obtain non-isomorphic Lie algebras $\mathcal{N}_{r, s}\left(U\left(p_{1}, q_{1}\right)\right)$ and $\mathcal{N}_{r, s}\left(U\left(p_{2}, q_{2}\right)\right)$ of the same dimension $R \cdot \operatorname{dim} V_{\text {min }}^{r, s ;+}$ if simultaneously $\left(p_{1}, q_{1}\right) \neq\left(p_{2}, q_{2}\right)$ and $\left(p_{1}, q_{1}\right) \neq$ $\left(q_{2}, p_{2}\right)$.

We fix an integer $k$ and determine all pairs of integers $\left(p_{i}, q_{i}\right)$ with the properties:

(a) $p_{i} \leq q_{i}$ for all $i$.

(b) $p_{i}+q_{i}=k$ for all $i$.

(c) $\left(p_{i}, q_{i}\right) \neq\left(p_{j}, q_{j}\right)$ and $\left(p_{i}, q_{i}\right) \neq\left(q_{j}, p_{j}\right)$ for $i \neq j$.

With such pairs we define:

$$
U_{i}:=\left(\bigoplus^{p_{i}} V_{\mathrm{min}}^{r, s ;+}\right) \bigoplus\left(\bigoplus^{q_{i}} V_{\mathrm{min}}^{r, s ;-}\right)
$$

From Theorem 7.5 and the above remark we conclude that the Lie algebras $\mathcal{N}_{r, s}\left(U_{i}\right)$ and $\mathcal{N}_{r, s}\left(U_{j}\right)$ are mutually non-isomorphic for $r \equiv 3 \bmod 4, s \equiv 1,2,3 \bmod 4$. In order to present a concrete family of nilmanifolds with the required properties we choose $r=3$ and $s=1$ such that $\operatorname{dim} V_{\min }^{3,1}=8$. Let $k$ be even and choose $m \in \mathbb{N}$ such that $k=2 m$. The pairs $\left(p_{i}, q_{i}\right)$ with (a)-(c) are of the form $\{(i, k-i) \mid 0 \leq i \leq m\}$ and we conclude:

Corollary 7.6 The following $m+1$ nilmanifolds

$$
\left(\Gamma_{3,1} \backslash \mathbb{G}_{3,1}\left(U_{i}\right)\right)_{0 \leq i \leq m}
$$

are isospectral, but mutually non-homeomorphic with respect to the (sub)-Laplacian.

Remark 7.7 For any given integer $m \in \mathbb{N}$ and by using the above method, we can construct $m+1$ nilmanifolds of the common dimension $4+16 m$ which are isospectral but mutually non-homeomorphic. In particular, one obtains a pair of such manifolds of the (minimal) dimension $4+16 \times 1=20$. Note that via the first method (i.e. Corollary 7.4) we can find a pair of such nilmanifolds of dimension 12 .

To minimize the dimension of the constructed family of nilmanifolds we should use a third method which is based on [15, Theorem 4.2], which treat the case $r \equiv 3 \bmod 4$ and $s \equiv 0 \bmod 4$. In this situation there are two non-equivalent irreducible representations and we use the lower index \pm in the notation below to distinguish the minimal admissible modules corresponding to each irreducible modules (or to each sum of irreducible modules, cf. Remark 4.1).

Theorem 7.8 (Furutani, Markina, [15]) For $r \equiv 3 \bmod 4$ and $s \equiv 0 \bmod 4$, let $U, \widetilde{U}$ be admissible modules decomposed into the direct sums:

$$
\begin{aligned}
& U=\left(\bigoplus^{p_{+}^{+}} V_{\text {min },+}^{r, s ;+}\right) \bigoplus\left(\bigoplus^{p_{+}^{-}} V_{\text {min },+}^{r, s ;-}\right) \bigoplus\left(\bigoplus^{p_{-}^{+}} V_{\text {min },-}^{r, s ;+}\right) \bigoplus\left(\bigoplus V_{\text {min,-- }}^{r, s ;-}\right), \\
& \tilde{U}=\left(\stackrel{\tilde{p}_{+}^{+}}{\bigoplus V_{\text {min },+}^{r, s ;+}}\right) \bigoplus\left(\stackrel{\tilde{p}_{+}^{-}}{\bigoplus V_{\text {min },+}^{r, s ;-}}\right) \bigoplus\left(\stackrel{\tilde{p}_{-}^{+}}{\bigoplus V_{\text {min },-}^{r, s ;+}}\right) \bigoplus\left(\stackrel{\tilde{p}_{-}^{-}}{\bigoplus} V_{\text {min,- }}^{r, s ;-}\right) .
\end{aligned}
$$


Then the Lie algebras $\mathcal{N}_{r, s}(U)$ and $\mathcal{N}_{r, s}(\widetilde{U})$ are isomorphic, if and only if one of the following conditions are fulfilled:

$$
\left[p_{+}^{+}+p_{-}^{-}=\tilde{p}_{+}^{+}+\tilde{p}_{-}^{-} \text {and } p_{+}^{-}+p_{-}^{+}=\tilde{p}_{+}^{-}+\tilde{p}_{-}^{+}\right]
$$

or

$$
\left[p_{+}^{+}+p_{-}^{-}=\tilde{p}_{+}^{-}+\tilde{p}_{-}^{+} \text {and } p_{+}^{-}+p_{-}^{+}=\tilde{p}_{+}^{+}+\tilde{p}_{-}^{-}\right] \text {. }
$$

To simplify the construction we choose $p_{-}^{+}=p_{-}^{-}=0, \widetilde{p}_{-}^{+}=\widetilde{p}_{-}^{-}=0$. The condition in Theorem 7.8 take the form:

$$
\left[p_{+}^{+}=\widetilde{p}_{+}^{+} \text {and } p_{+}^{-}=\tilde{p}_{+}^{-}\right] \text {or }\left[p_{+}^{+}=\tilde{p}_{+}^{-} \text {and } p_{+}^{-}=\tilde{p}_{+}^{+}\right] .
$$

Next, we choose $r=3, s=0$ and fix $m \in \mathbb{N}$. Then we consider the following family of $m+1$ admissible modules:

$$
V_{i}:=\left(\bigoplus^{i} V_{\text {min;+ }}^{3,0 ;+}\right) \bigoplus\left(\bigoplus^{2 m-i} V_{\text {min;+ }}^{3,0 ;-}\right), \quad(0 \leq i \leq m) .
$$

We obtain a family of $m+1$ mutually non-homeomorphic, isospectral nilmanifolds of common dimension $3+8 m$.

Corollary 7.9 For $0 \leq i \leq m$, the $m+1$ nilmanifolds

$$
\left(\Gamma_{3,0} \backslash \mathbb{G}_{3,0}\left(V_{i}\right)\right)_{0 \leq i \leq m}
$$

are isospectral but mutually non-homeomorphic.

Remark 7.10 By choosing $m=1$ we obtain a pair of nilmanifolds both having dimension $3+8=11$. This dimension is minimal among the previous examples.

\section{Subriemannian structure and heat trace expansion}

To every pseudo $H$-type nilmanifold $M=\Gamma_{r, s} \backslash \mathbb{G}_{r, s}$ with $r+s>1$ and based on [3, Theorem 3.3] we construct a Heisenberg manifold $H=\Gamma \backslash \mathbb{H}_{2 n+1}$ such that the short time heat trace asymptotic expansions corresponding to the sub-Laplacians on $M$ and $H$, respectively, coincide up to a term vanishing to infinite order. Moreover, in our construction the manifolds $M$ and $H_{\alpha}$ have different dimensions. Recall that in the case of a Riemannian structure on $M$ the heat trace expansion corresponding to the Laplacian encodes the dimension of $M$ and therefore such examples do not exist in the framework of Riemannian geometry.

Let $d=r+s>1$, and with our previous notation consider the nilmanifold

$$
M=\Gamma_{r, s} \backslash \mathbb{G}_{r, s} \text { with } \operatorname{dim} M=2 N+d .
$$

We write $\mathbb{H}_{2 n+1}=\mathbb{G}_{1,0}\left(\mathbb{R}^{2 n}\right)$ for the $(2 n+1)$-dimensional Heisenberg group and with $\alpha>0$ we define a lattice $\Gamma_{\alpha} \subset \mathbb{H}_{2 n+1}$ of the form:

$$
\Gamma_{\alpha}=\left\{\sqrt{\alpha} \sum m_{i} X_{i}+\frac{\alpha}{2} k Z: m_{i}, k \in \mathbb{Z}\right\} .
$$

Here $\left\{X_{i}, Z \mid i=1, \ldots, 2 n\right\}$ denotes a basis of the Lie algebra of $\mathbb{H}_{2 n+1}$ with the non-trivial bracket relations

$$
\left[X_{i}, X_{n+j}\right]=\delta_{i j} Z, \quad(i, j=1, \ldots, n) .
$$


The corresponding one-parameter family of Heisenberg manifolds will be denoted by:

$$
H_{\alpha}=\Gamma_{\alpha} \backslash \mathbb{H}_{2 n+1} \text { where } \alpha>0 .
$$

We recall the form of the short time heat trace asymptotic expansion of the sub-Laplacian on a general compact 2-step nilmanifold $\Gamma \backslash \mathbb{G}$ in [3, Theorem 3.3]:

Theorem 8.1 (Bauer, Furutani, Iwasaki [3]) Let $M=\Gamma \backslash \mathbb{G}$ be a 2-step compact nilmanifold of dimension $2 N+d$. Then

$$
\operatorname{tr}\left(e^{-t \Delta_{\text {sub }}^{M}}\right)=\frac{c_{M}}{t^{N+d}}+O\left(t^{\infty}\right) \text { as } t \rightarrow 0 .
$$

The constant $c_{M}>0$ explicitly is given by

$$
c_{M}=\frac{\operatorname{Vol}(M)}{(2 \pi)^{N+d}} \int_{\mathbb{R}^{d}} W(\tau) d \tau .
$$

If we apply the above theorem to $H_{\alpha}$, we obtain:

$$
c_{H_{\alpha}}=\frac{\operatorname{Vol}\left(H_{\alpha}\right)}{(2 \pi)^{n+1}} \int_{\mathbb{R}} W(\tau) d \tau .
$$

Here $W(\tau) d \tau$ is simply the volume form in Theorem 2.2 and associated to the Heisenberg group with the above structure constants. Note that

$$
\operatorname{Vol}\left(H_{\alpha}\right)=\alpha^{n+1} \operatorname{Vol}\left(H_{1}\right) .
$$

If we choose $n \in \mathbb{N}$ with $n+1=N+d$ and $\alpha>0$ such that $c_{H_{\alpha}}=c_{M}$, then we obtain a pair of compact, subriemannian manifolds with the properties:

(a) $\operatorname{tr}\left(e^{-t \Delta_{\text {sub }}^{M}}\right)-\operatorname{tr}\left(e^{-t \Delta_{\text {sub }}^{H_{\alpha}}}\right)=O\left(t^{\infty}\right)$ as $t \rightarrow 0$

(b) $2 N+d=\operatorname{dim} M \neq \operatorname{dim} H_{\alpha}=2 N+2 d-1$ (since $d>1$ ).

Remark 8.2 From the heat trace expansion for small times we can read the Hausdorff dimension $2(N+d)$ of the nilmanifold $M=\Gamma \backslash \mathbb{G}$ in Theorem 8.1 considered as a metric space with respect to the Carnot-Carathéodory distance. However, the last example indicates that we cannot read the Euclidean dimension of $M$ from the coefficient (8.1) of the heat trace expansion. However, in Remark 3.2 we have pointed out that in some cases this dimension can be obtained from the full spectrum of the sub-Laplacian. In the case of the nilmanifolds $M$ in this paper and which are constructed from a standard lattice we have $\operatorname{vol}(M)=1$ and therefore, with the notation in Theorem 8.1:

$$
c_{M}=C_{M}(N, d):=\frac{1}{(2 \pi)^{N+d}} \int_{\mathbb{R}^{d}} W(\tau) d \tau .
$$

We list a few problems concerning the geometric information contained in the spectrum of the sub-Laplacian on a nilmanifold:

\section{Problems:}

(a) Let $\mathbb{G}_{r, s}(V)$ be a pseudo $H$-type group with standard lattice $\Gamma_{r, s}$ as explained in Section 4. Can we determine the numbers $2 N=\operatorname{dim} V$ and $d=\operatorname{dim} \mathbb{G}_{r, s}(V)-2 N$ from the coefficient (8.2)?

Consider the case $s=0$. Then integration with respect to polar coordinates shows:

$$
\int_{\mathbb{R}^{d}} W(\tau) d \tau=\int_{\mathbb{R}^{d}} \frac{|\tau|^{N}}{(\sinh |\tau|)^{N}} d \tau=2 V_{d} \int_{0}^{\infty} \frac{r^{N+d-1} e^{-N r}}{\left(1-e^{-2 r}\right)^{N}} d r=(*),
$$


where $V_{d}=2 \pi^{\frac{d}{2}} / \Gamma\left(\frac{d}{2}\right)$ denotes the volume of the $(d-1)$-dimensional unit sphere. Now we use the following power series expansion for $|x|<1$ :

$$
\frac{1}{(1-x)^{N}}=\sum_{\alpha \in \mathbb{N}_{0}^{N}} x^{|\alpha|}
$$

A change of variables in the integral is applied to obtain:

$$
\begin{aligned}
(*) & =2 V_{d} \sum_{\alpha \in \mathbb{N}_{0}^{N}} \int_{0}^{\infty} r^{N+d-1} e^{-(N+2|\alpha|) r} d r \\
& =2 V_{d} \sum_{\alpha \in \mathbb{N}_{0}^{N}} \frac{1}{(N+2|\alpha|)^{N+d}} \int_{0}^{\infty} r^{N+d-1} e^{-r} d r \\
& =\frac{V_{d} \Gamma(N+d)}{2^{N+d-1}} \sum_{\alpha \in \mathbb{N}_{0}^{N}} \frac{1}{\left(\frac{N}{2}+|\alpha|\right)^{N+d}} \\
& =\frac{V_{d} \Gamma(N+d)}{2^{N+d-1}} \zeta_{N}\left(N+d, \frac{N}{2}\right) .
\end{aligned}
$$

The infinite sum is called multiple Hurwitz zeta function and previously has been studied in the literature:

$$
\zeta_{N}\left(N+d, \frac{N}{2}\right):=\sum_{\alpha \in \mathbb{N}_{0}^{N}} \frac{1}{\left(\frac{N}{2}+|\alpha|\right)^{N+d}} .
$$

Hence:

$$
c_{M}(N, d)(2 \pi)^{N+d} \frac{2^{N+d-2}}{\Gamma(N+d)}=\frac{\pi^{\frac{d}{2}}}{\Gamma\left(\frac{d}{2}\right)} \zeta_{N}\left(N+d, \frac{N}{2}\right) .
$$

The left hand side can be calculated from the spectral data (more precisely, from the heat trace expansion in Theorem 8.1). Hence the problem reduces to the question, whether for each $k \in \mathbb{N}$ the assignment:

$$
N_{k}:=\left\{(N, d) \in \mathbb{N}^{2}: N+d=k\right\} \ni(N, d) \mapsto \frac{\pi^{\frac{d}{2}}}{\Gamma\left(\frac{d}{2}\right)} \zeta_{N}\left(k, \frac{N}{2}\right)
$$

is injective.

(b) Consider two isospectral compact nilmanifolds $M_{j}=\Gamma_{j} \backslash G_{j}$ where $j=1,2$. Assume that both are equipped with a left-invariant subriemannian structure as described in this paper. Is it true that $\operatorname{dim} G_{1}=\operatorname{dim} G_{2}$ (see Remark 6.1)?

(c) The distribution of eigenvalues for classes of hypoelliptic operators with double characteristics on compact manifolds and under additional conditions is well-studied (e.g. see the work by Menikoff and Sjöstrand [29-31]), and Melrose [28]. Moreover, in the case of "sum-of-squares operators" satisfying Hörmander's bracket generating condition the asymptotic of the heat kernel at small times was found by Ben Arous, Léandre (see $[7,8]$ ) in a form which encodes geometric data of an induced subriemannian structure (such as the Carnot Carathéodory metric). However, not much seems to be known on the precise growth order or coefficient of the second term in the expansion of the eigenvalue counting function for the sub-Laplacian on compact nilmanifolds. Based on a classification of lattices and the explicit spectral data such question in the case of Heisenberg manifolds has been discussed in [36]. In generalizing R. Strichartz's 
result one may study the following problem:

Let $M:=\Gamma \backslash G$ denote a compact nilmanifold (e.g. modelled over a pseudo $H$-type Lie group). Determine the growth order or even the coefficient of the second term in the eigenvalue counting function for the corresponding sub-Laplacian.

Acknowledgements Open Access funding provided by Projekt DEAL.

Open Access This article is licensed under a Creative Commons Attribution 4.0 International License, which permits use, sharing, adaptation, distribution and reproduction in any medium or format, as long as you give appropriate credit to the original author(s) and the source, provide a link to the Creative Commons licence, and indicate if changes were made. The images or other third party material in this article are included in the article's Creative Commons licence, unless indicated otherwise in a credit line to the material. If material is not included in the article's Creative Commons licence and your intended use is not permitted by statutory regulation or exceeds the permitted use, you will need to obtain permission directly from the copyright holder. To view a copy of this licence, visit http://creativecommons.org/licenses/by/4.0/.

\section{Appendix}

In the appendix we present the dimensions of minimal admissible modules for some basic cases in Table 2. These data are taken from [14,15] which we refer to for more details and notations. The remaining cases can be obtained by $(4,4),(8,0)$ and $(0,8)$-periodicities with respect to the signature $(r, s)$, respectively. In particular, the table indicates the cases in which two non-equivalent minimal admissible modules exist. However, it is known that pseudo $H$-type algebras constructed from two non-equivalent minimal admissible modules are isomorphic.

Table 2 Dimensions of minimal admissible modules

\begin{tabular}{llllllllll}
\hline 8 & $16^{ \pm}$ & & & & & & & \\
7 & $16^{N}$ & $32^{N}$ & $\mathbf{6 4}^{N}$ & $64^{ \pm}$ & & & & \\
6 & $16^{N}$ & $16_{\times 2}^{N}$ & $32^{N}$ & $32^{ \pm}$ & & & & \\
5 & $\mathbf{1 6}^{N}$ & $16^{N}$ & $16^{N}$ & $16^{ \pm}$ & & & & \\
4 & $8^{ \pm}$ & $8^{ \pm}$ & $8^{ \pm}$ & $8_{\times 2}^{ \pm}$ & $16^{ \pm}$ & & & & \\
3 & $\mathbf{8}^{N}$ & $8^{N}$ & $8^{N}$ & $8^{ \pm}$ & $16^{N}$ & $32^{N}$ & $\mathbf{6 4}^{N}$ & $64^{ \pm}$ & \\
2 & $\mathbf{4}^{N}$ & $4_{\times 2}^{N}$ & $\mathbf{8}^{N}$ & $8^{ \pm}$ & $16^{N}$ & $16_{\times 2}^{N}$ & $32^{N}$ & $32^{ \pm}$ & \\
1 & $2^{N}$ & $4^{N}$ & $\mathbf{8}^{N}$ & $8^{ \pm}$ & $\mathbf{1 6}^{N}$ & $16^{N}$ & $16^{N}$ & $16^{ \pm}$ & \\
0 & $1^{ \pm}$ & $2^{ \pm}$ & $4^{ \pm}$ & $4_{\times 2}^{ \pm}$ & $8^{ \pm}$ & $8^{ \pm}$ & $8^{ \pm}$ & $8_{\times 2}^{ \pm}$ & $16^{ \pm}$ \\
$\mathrm{s} / \mathrm{r}$ & 0 & 1 & 2 & 3 & 4 & 5 & 6 & 7 & 8 \\
\hline
\end{tabular}

black $=$ irreducible, bold $=$ double of irreducible,

$* \times 2=$ two non-equivalent minimal dimensional admissible modules

\section{References}

1. Agrachev, A., Boscain, U., Gauthier, J.-P., Rossi, F.: The intrinsic hypoelliptic Laplacian and its heat kernel on unimodular Lie groups. J. Funct. Anal. 256, 2621-2655 (2009)

2. Atiyah, M.F., Bott, R., Shapiro, A.: Clifford modules. Topology 3(Suppl. I), 3-38 (1964) 
3. Bauer, W., Furutani, K., Iwasaki, C.: Spectral zeta function of the sub-Laplacian on two step nilmanifolds. J. Math. Pures Appl. (9) 97(3), 242-261 (2012)

4. Bauer, W., Furutani, K., Iwasaki, C.: Spectral zeta function on pseudo $H$-type nilmanifolds. Indian J. Pure Appl. Math. 46(4), 539-582 (2015)

5. Beals, R., Gaveau, B., Greiner, P.: The Green function of model step two hypoelliptic operators and the analysis of certain tangential Cauchy Riemannian complexes. Adv. Math. 121, 288-345 (1996)

6. Beals, R., Gaveau, B., Greiner, P.: Hamilton-Jacobi theory and the heat kernel on Heisenberg groups. J. Math. Pures Appl. 79(7), 633-689 (2000)

7. Ben Arous, G.: Développement asymptotique du noyau de la chaleur hypoelliptique sur la diagonale (French). Ann. Inst. Fourier (Grenoble) 39(1), 73-99 (1989)

8. Ben Arous, G., Léandre, R.: Décraissance exponentielle du noyau de la chaleur sur la diagonale I + II (French). Probab. Theory Relat. Fields 90(2), 175-202 (1991). (no. 3, 377-402)

9. Calin, O., Chang, D.-C., Furutani, K., Iwasaki, C.: Heat Kernels for Elliptic and Sub-elliptic Operators Methods and Techniques. Applied and Numerical Harmonic Analysis. Birkhäuser/Springer, New York (2011)

10. Ciatti, P.: Scalar products on Clifford modules and pseudo-H-type Lie algebras. Ann. Mat. Pura Appl. (4) 178, 1-31 (2000)

11. Crandall, G., Dodziuk, J.: Integral structures on H-type Lie algebras. J. Lie Theory 12(1), 69-79 (2002)

12. Furutani, K.: Heat kernels of the sub-Laplacian and Laplacian on nilpotent Lie groups. In: Wojciechowsky, P. (ed.) Analysis, Geometry and Topology of Elliptic Operators, Papers in Honor of Krzysztof, pp. 185226. World Scientific, London (2006)

13. Furutani, K., Markina, I.: Existence of lattices on general $H$-type groups. J. Lie Theory 24, 979-1011 (2014)

14. Furutani, K., Markina, I.: Complete classification of pseudo H-type algebras: I. Geom. Dedicata 190, 23-51 (2017)

15. Furutani, K., Markina, I.: Complete classification of pseudo H-type algebras: II. Geom. Dedicata 202, 233-264 (2019)

16. Gordon, C., Webb, D., Welpert, S.: Isospectral plane domains and surfaces via Riemannian orbifolds. Invent. Math. 110(1), 1-22 (1992)

17. Gorneth, R.: A new construction of isospectral Riemannian nilmanifolds with examples. Mich. Math. J. 43, 159-188 (1996)

18. Hörmander, L.: Hypo-elliptic second order differential equations. Acta Math. 119, 147-171 (1967)

19. Ikeda, A.: On lens spaces which are isospectral but not isometric. Ann. Sci. École Norm Sup. (4) 13(3), 303-315 (1980)

20. Ikeda, A.: On spherical space forms which are isospectral but not isometric. J. Math. Soc. Jpn. 35, 437-444 (1983)

21. Iwasaki, C., Iwasaki, N.: Parametrix for a degenerate parabolic equation and its application to the asymptotic behavior of spectral functions for stationary problems. Publ. Res. Inst. Math. Sci. 17(2), 577-655 (1981)

22. Kac, M.: Can one hear the shape of a drum? Am. Math. Mon. 73(4 part II), 1-23 (1966)

23. Kaplan, A.: Fundamental solutions for a class of hypoelliptic PDE generated by composition of quadratic forms. Trans. Am. Math. Soc. 258, 147-153 (1980)

24. Kaplan, A.: On the geometry of groups of Heisenberg type. Bull. Lond. Math. Soc. 15(1), 35-42 (1983)

25. Lawson, H.B., Michelson, M.-L.: Spin Geometry. Princeton University Press, Princeton (1989)

26. Malćev, A.I.: On a class of homogeneous spaces, Amer. Math. Soc. Translation 39 (1951) (Izv. Akad. Nauk USSR, Ser. Mat. 13, 9-32 (1949))

27. Mckean, H.P.: Selberg's trace formula as applied to a compact surface. Commun. Pure Appl. Math. 25, 225-246 (1972)

28. Melrose, R.B.: The wave equation for a hypoelliptic operator with symplectic characteristics of codimension two. J. Anal. Math. 44(1), 134-182 (1984)

29. Menikoff, A., Sjöstrand, J.: On the eigenvalue of a class of hypoelliptic operators. II. Global analysis (Proc. Biennial Sem. Canad. Math. Congr., Univ. Calgary, Alta. 1978), Lecture Notes in Math. 755, Springer, Berlin (1979)

30. Menikoff, A., Sjöstrand, J.: On the eigenvalues of a class of hypoelliptic operators. Math. Ann. 235(1), 55-85 (1978)

31. Menikoff, A., Sjöstrand, J.: The eigenvalues of hypoelliptic operators. III. The nonsemibounded case. J. Anal. Math. 35, 123-150 (1979)

32. Milnor, J.: Eigenvalues of the Laplace operator on certain manifolds. Proc. Nat. Acad. Sci. USA 51, 542 (1964)

33. Raghunathan, M.S.: Discrete Subgroups of Lie Groups. Springer, New York (1972) 
34. Strichartz, R.S.: Sub-Riemannian geometry. J. Differ. Geom. 24, 221-263 (1986)

35. Strichartz, R.S.: Corrections to "Sub-Riemannian geometry". J. Differ. Geom. 30, 595-596 (1989)

36. Strichartz, R.S.: Spectral Asymptotics on Compact Heisenberg Manifolds. J. Geom. Anal. 26(3), 24502458 (2016)

37. Sunada, T.: Riemannian coverings and isospectral manifolds. Ann. Math. 121(2), 169-186 (1985)

Publisher's Note Springer Nature remains neutral with regard to jurisdictional claims in published maps and institutional affiliations. 